\title{
On characterizations for subclasses of directed co-graphs
}

\author{
Frank Gurski $^{1}\left[\right.$ Dominique Komander $^{1} \cdot$ Carolin Rehs $^{1}$
}

Accepted: 1 November 2020 / Published online: 27 November 2020

(c) The Author(s) 2020

\begin{abstract}
Undirected co-graphs are those graphs which can be generated from the single vertex graph by disjoint union and join operations. Co-graphs are exactly the $P_{4}$-free graphs (where $P_{4}$ denotes the path on 4 vertices). The class of co-graphs itself and several subclasses haven been intensively studied. Among these are trivially perfect graphs, threshold graphs, weakly quasi threshold graphs, and simple co-graphs. Directed cographs are digraphs which can be defined by, starting with the single vertex graph, applying the disjoint union, order composition, and series composition. By omitting the series composition we obtain the subclass of oriented co-graphs which has been analyzed by Lawler in the 1970s. The restriction to linear expressions was recently studied by Boeckner. Until now, there are only a few versions of subclasses of directed co-graphs. By transmitting the restrictions of undirected subclasses to the directed classes, we define the corresponding subclasses for directed co-graphs. We consider directed and oriented versions of threshold graphs, simple co-graphs, co-simple cographs, trivially perfect graphs, co-trivially perfect graphs, weakly quasi threshold graphs and co-weakly quasi threshold graphs. For all these classes we give characterizations by finite sets of minimal forbidden induced subdigraphs. Additionally, we analyze the relations between these graph classes.
\end{abstract}

Keywords Co-graphs · Directed co-graphs · Directed threshold graphs · Digraphs · Threshold graphs $\cdot$ Forbidden induced subdigraph characterizations

An extended abstract of this paper appeared in the Proceedings of the International Conference on Combinatorial Optimization and Applications (COCOA 2019; see Gurski et al. 2019a).

Frank Gurski

frank.gurski@hhu.de

Dominique Komander

dominique.komander@hhu.de

Carolin Rehs

carolin.rehs@hhu.de

1 Institute of Computer Science, Algorithmics for Hard Problems Group, University of Düsseldorf, 40225 Düsseldorf, Germany 


\section{Introduction}

During the last years classes of directed graphs have received a lot of attention (BangJensen and Gutin 2018) since they are useful in multiple applications of directed networks. For instance the class of directed co-graphs can be used in applications in the field of genetics (see Nojgaard et al. 2018). However, the field of directed co-graphs is far less well studied than the undirected version, although it has a similarly useful structure. There are multiple subclasses of undirected co-graphs which are already characterized successfully by several definitions. Among these are trivially perfect graphs (Golumbic 1978), threshold graphs (Chvátal and Hammer 1977), weakly quasi threshold graphs (Bapat et al. 2008; Nikolopoulos and Papadopoulos 2011), and simple co-graphs (Heggernes et al. 2011), see Table 2. Moreover, there are also corresponding subclasses of directed co-graphs such as e.g. the class of oriented co-graphs, which has been analyzed by Lawler (1976) and Boeckner (2018). But there are many more interesting subclasses of directed co-graphs, that are mostly not characterized until now. To close this gap we consider directed versions of threshold graphs, simple cographs, trivially perfect graphs and weakly quasi threshold graphs, of which each class is structurally different and interesting. To obtain completeness we take a look at the oriented versions of these classes and the related complement classes. Further, in order to get an overview of the classes, we compare the classes to each other and visualize the relations between them. All of these classes are hereditary, just like directed cographs, such that they can be characterized by a set of forbidden induced subdigraphs. We can even show a finite number of forbidden induced subdigraphs for the classes, that are introduced in the following. This property is very useful, for example in the case of finding an polynomial recognition algorithm for these classes.

Undirected co-graphs, i.e. complement reducible graphs, appeared independently by several authors (see Lerchs 1971; Sumner 1974) for example, while directed cographs were introduced 30 years later by Bechet et al. (1997). Due to their recursive structure there are problems, that are hard in general, which can be solved efficiently on (directed) co-graphs (see Bodlaender and Möhring 1993; Corneil et al. 1981, 1984; Lin et al. 1995; Gurski et al. 2020b; Bang-Jensen and Maddaloni 2014; Gurski 2017; Gurski et al. 2019b, c; Gurski and Rehs 2018b; Gurski et al. 2020a,c). That makes both graph classes particularly interesting. This paper can be seen a basis for further research on possible algorithms that are efficient for the structures of the presented graph classes.

This paper is organized as follows. After introducing some basic definitions we introduce undirected co-graphs in Sect. 3 and subclasses. Moreover, we recapitulate their relations and their characterizations by sets of forbidden subgraphs. In Sect. 4 we introduce directed and oriented co-graphs and summarize their properties. Subsequently, we focus on subclasses of directed co-graphs. We show definitions of series-parallel partial order digraphs, directed trivially perfect graphs, directed weakly quasi threshold graphs, directed simple co-graphs, directed threshold graphs and the corresponding complementary and oriented versions of these classes. Some of the subclasses already exist, others are motivated by the alike subclasses of undirected co-graphs, see Table 2 . All of these subclasses can be constructed recursively by several operations. Analogously to the undirected classes, we show how these multiple 
subclasses can be characterized by finite sets of minimal forbidden induced subdigraphs. We continue with an analysis of the relations of the several classes. Moreover, we examine how they are related to the corresponding undirected classes. Finally in Sect. 5, we give conclusions including further research directions.

\section{Preliminaries}

\subsection{Notations for undirected graphs}

We work with finite undirected graphs $G=(V, E)$, where $V$ is a finite set of vertices and $E \subseteq\{\{u, v\} \mid u, v \in V, u \neq v\}$ is a finite set of edges. A graph $G^{\prime}=\left(V^{\prime}, E^{\prime}\right)$ is a subgraph of graph $G=(V, E)$ if $V^{\prime} \subseteq V$ and $E^{\prime} \subseteq E$. If every edge of $E$ with both end vertices in $V^{\prime}$ is in $E^{\prime}$, we say that $G^{\prime}$ is an induced subgraph of digraph $G$ and we write $G^{\prime}=G\left[V^{\prime}\right]$.

For a graph $G=(V, E)$ its complement graph is defined by

$$
\operatorname{co}-G=(V,\{\{u, v\} \mid\{u, v\} \notin E, u, v \in V, u \neq v\})
$$

For a graph class $X$ we define by co- $X=\{$ co- $G \mid G \in X\}$.

For graph $G$ and an integer $d$ let $d G$ be the disjoint union of $k$ copies of $G$. Special undirected graphs As usual we denote by

$$
K_{n}=\left(\left\{v_{1}, \ldots, v_{n}\right\},\left\{\left\{v_{i}, v_{j}\right\} \mid 1 \leq i<j \leq n\right\}\right),
$$

$n \geq 1$ a complete graph on $n$ vertices and by $I_{n}$ an edgeless graph on $n$ vertices, i.e. the complement graph of a complete graph on $n$ vertices. By

$$
P_{n}=\left(\left\{v_{1}, \ldots, v_{n}\right\},\left\{\left\{v_{1}, v_{2}\right\}, \ldots,\left\{v_{n-1}, v_{n}\right\}\right\}\right)
$$

we denote a path on $n$ vertices. See Table 1 for examples.

\subsection{Notations for directed graphs}

A directed graph or digraph is a pair $G=(V, E)$, where $V$ is a finite set of vertices and $E \subseteq\{(u, v) \mid u, v \in V, u \neq v\}$ is a finite set of ordered pairs of distinct ${ }^{1}$ vertices called arcs. A vertex $v \in V$ is out-dominating (in-dominated) if it is adjacent to every other vertex in $V$ and is a source (a sink, respectively). A digraph $G^{\prime}=\left(V^{\prime}, E^{\prime}\right)$ is a subdigraph of digraph $G=(V, E)$ if $V^{\prime} \subseteq V$ and $E^{\prime} \subseteq E$. If every arc of $E$ with both end vertices in $V^{\prime}$ is in $E^{\prime}$, we say that $G^{\prime}$ is an induced subdigraph of digraph $G$ and we write $G^{\prime}=G\left[V^{\prime}\right]$.

For a directed graph $G=(V, E)$ its complement digraph is defined by

$$
\operatorname{co}-G=(V,\{(u, v) \mid(u, v) \notin E, u, v \in V, u \neq v\})
$$

\footnotetext{
1 Thus, we do not consider directed graphs with loops or multiple edges.
} 
and its converse digraph is defined by

$$
G^{c}=(V,\{(u, v) \mid(v, u) \in E, u, v \in V, u \neq v\}) .
$$

For a digraph class $X$ we define by co- $X=\{$ co- $G \mid G \in X\}$. For a digraph $G$ and an integer $d$ let $d G$ be the disjoint union of $k$ copies of $G$.

Orientations There are several ways to define a digraph $D=(V, A)$ from an undirected graph $G=(V, E)$, see (Bang-Jensen and Gutin 2009). If we replace every edge $\{u, v\}$ of $G$ by

- one of the $\operatorname{arcs}(u, v)$ and $(v, u)$, we denote $D$ as an orientation of $G$. Every digraph $D$ that can be obtained by an orientation of an undirected graph $G$ is called an oriented graph.

- one or both of the $\operatorname{arcs}(u, v)$ and $(v, u)$, we denote $D$ as a biorientation of $G$. A digraph $D$ that we get by a biorientation of an undirected graph $G$ is called a bioriented graph.

- both $\operatorname{arcs}(u, v)$ and $(v, u)$, we denote $D$ as a complete biorientation of $G$. Since in this case $D$ is well defined by $G$ we also denote it by $\overleftrightarrow{G}$. A digraph $D$ that we obtain by a complete biorientation of an undirected graph $G$ is called a complete bioriented graph.

For a given digraph $D=(V, A)$ we define its underlying undirected graph by ignoring the directions of the edges and deleting multiple edges, i.e.

$$
\operatorname{und}(D)=(V,\{\{u, v\} \mid(u, v) \in A, u, v \in V\}) .
$$

and for some class of digraphs $X$, let

$$
\operatorname{und}(X)=\{\operatorname{und}(D) \mid D \in X\} .
$$

Special Directed Graphs As usual we denote by

$$
\overleftrightarrow{K_{n}}=\left(\left\{v_{1}, \ldots, v_{n}\right\},\left\{\left(v_{i}, v_{j}\right) \mid 1 \leq i \neq j \leq n\right\}\right)
$$

$n \geq 1$ a bidirectional complete digraph on $n$ vertices and by $\overleftrightarrow{I_{n}}$ an edgeless graph with $n$ vertices, i.e. the complement graph of a complete directed graph on $n$ vertices. By

$$
\overleftrightarrow{K_{n, m}}=\left(\left\{v_{1}, \ldots, v_{n}, w_{1}, \ldots, w_{m}\right\},\left\{\left(v_{i}, w_{j}\right),\left(w_{j}, v_{i}\right) \mid 1 \leq i \leq n, 1 \leq j \leq m\right\}\right)
$$

$n, m \geq 1$ a bidirectional complete bipartite digraph with $n+m$ vertices. A directed clique is a bidirectional complete digraph, such that $K=(V, E)$ with $E=\{(u, v) \mid$ $u, v \in V, u \neq v\}$ holds.

Special oriented graphs By

$$
\overrightarrow{P_{n}}=\left(\left\{v_{1}, \ldots, v_{n}\right\},\left\{\left(v_{1}, v_{2}\right), \ldots,\left(v_{n-1}, v_{n}\right)\right\}\right)
$$


we denote the oriented path on $n$ vertices. By

$$
\overrightarrow{C_{n}}=\left(\left\{v_{1}, \ldots, v_{n}\right\},\left\{\left(v_{1}, v_{2}\right), \ldots,\left(v_{n-1}, v_{n}\right),\left(v_{n}, v_{1}\right)\right\}\right)
$$

we denote the oriented cycle on $n$ vertices. By $T_{n}$ we denote a transitive tournament on $n$ vertices. $^{2}$ By

$$
\overrightarrow{K_{n, m}}=\left(\left\{v_{1}, \ldots, v_{n}, w_{1}, \ldots, w_{m}\right\},\left\{\left(v_{i}, w_{j}\right) \mid 1 \leq i \leq n, 1 \leq j \leq m\right\}\right),
$$

$n, m \geq 1$ we denote an oriented complete bipartite digraph on $n+m$ vertices.

\subsection{Induced subgraph characterizations for hereditary classes}

The notations and results below are given in Kitaev and Lozin (2015, Chapter 2) for undirected graphs. These results can be transferred, as they also hold for directed graphs.

If classes of (di)graphs are closed under taking induced sub(di)graphs, they are called hereditary. For a (di)graph class $F$ we define Free $(F)$ as the set of all (di)graphs $G$ such that no induced sub(di)graph of $G$ is isomorphic to a (di)graph in set $F$.

Theorem 1 (Kitaev and Lozin 2015) Let X be a class of (di)graphs. $X$ is hereditary if and only if there is a set $F$ for which holds that Free $(F)=X$.

A (di)graph $G$ is a minimal forbidden induced sub(di)graph for a hereditary class $X$ if $G$ does not belong to $X$ and if every proper induced sub(di)graph of $G$ is a member of $X$. For a hereditary (di)graph class $X$ let $\operatorname{Forb}(X)$ be the set of all minimal forbidden induced sub(di)graphs of $X$.

Theorem 2 (Kitaev and Lozin 2015) For a hereditary class of (di)graphs $X$ it holds that $X=$ Free $(F o r b(X))$, where the set Forb $(X)$ is unique and of minimum size.

Theorem 3 (Kitaev and Lozin 2015) I holds that Free $\left(F_{1}\right) \subseteq$ Free $\left(F_{2}\right)$ if and only if for every (di)graph $G \in F_{2}$ it exists a (di)graph $H \in F_{1}$ with $H$ is an induced sub(di)graph of $G$.

Lemma 1 (Kitaev and Lozin 2015) Let $X=\operatorname{Free}\left(F_{1}\right)$ and $Y=\operatorname{Free}\left(F_{2}\right)$ be some hereditary classes of (di)graphs. Then, it holds that $X \cap Y=\operatorname{Free}\left(F_{1} \cup F_{2}\right)$ and co- $X=$ Free $\left(\right.$ co- $\left.F_{1}\right)$.

Observation 1 Let $G$ be a digraph with $G \in$ Free $(X)$ for a hereditary class of digraphs Free $(X)$ and it exists a digraph $X^{*} \in X$ such that every biorientation of und $\left(X^{*}\right)$ is in Free $(X)$, then it holds that und $(G) \in$ Free $\left(\right.$ und $\left.\left(X^{*}\right)\right)$.

Observation 2 Let $G$ be a digraph such that und $(G) \in$ Free $(X)$ for some hereditary class of graphs Free $(X)$, then for all $X^{*} \in X$ and all biorientations $b\left(X^{*}\right)$ of $X^{*}$ it holds that $G \in \operatorname{Free}\left(b\left(X^{*}\right)\right)$.

\footnotetext{
2 Please note that transitive tournaments on $n$ vertices are unique up to isomorphism (see Gould 2012, Chapter 9), Gurski et al. (2018) and Lemma 5.
} 
Table 1 Special graphs

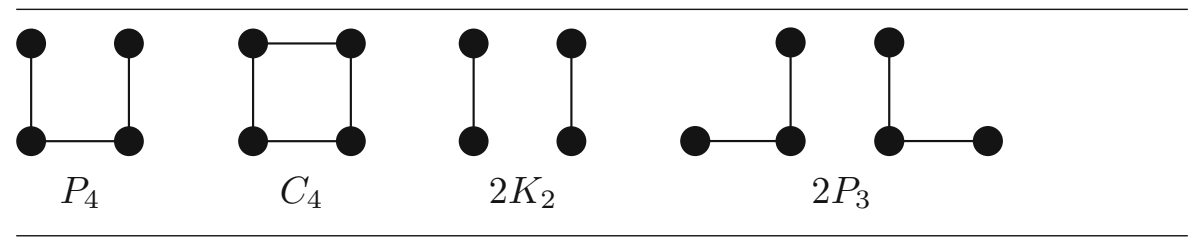

\section{Undirected co-graphs and subclasses}

For the definition of co-graphs and subclasses we use the following operations. Let $G_{1}=\left(V_{1}, E_{1}\right)$ and $G_{2}=\left(V_{2}, E_{2}\right)$ be two vertex-disjoint graphs.

- The disjoint union of $G_{1}$ and $G_{2}$, denoted by $G_{1} \oplus G_{2}$, is the graph with vertex set $V_{1} \cup V_{2}$ and edge set $E_{1} \cup E_{2}$.

- The join of $G_{1}$ and $G_{2}$, denoted by $G_{1} \otimes G_{2}$, is the graph with vertex set $V_{1} \cup V_{2}$ and edge set $E_{1} \cup E_{2} \cup\left\{\left\{v_{1}, v_{2}\right\} \mid v_{1} \in V_{1}, v_{2} \in V_{2}\right\}$.

We also recall the forbidden induced subgraph characterizations for co-graphs and frequently analyzed subclasses. Therefore, the graphs in Table 1 are very useful.

\subsection{Co-graphs}

Definition 1 (Co-graphs Corneil et al. 1981) The class of co-graphs (short for complement-reducible graphs) is defined recursively as follows.

(i) A graph on a single vertex $(\{v\}, \emptyset)$, denoted by $\bullet$, is a co-graph.

(ii) If $G_{1}$ and $G_{2}$ are co-graphs, then (a) $G_{1} \oplus G_{2}$ and (b) $G_{1} \otimes G_{2}$ are co-graphs.

We denote the class of co-graphs by $\mathrm{C}$.

Using the recursive structure of co-graphs many problems can be solved in linear time (e.g. see Corneil et al. 1981). Further, the recursive structure allows us to compute the path-width, as well as the tree-width of co-graphs in linear time (Bodlaender and Möhring 1993).

\subsection{Subclasses of co-graphs}

In Table 2 we summarize co-graphs and their well-known subclasses. The given forbidden sets are known from the existing literature (Corneil et al. 1981; Golumbic 1978; Chvátal and Hammer 1977; Nikolopoulos and Papadopoulos 2011; Heggernes et al. 2011).

\subsection{Relations}

In Fig. 1 we compare the above graph classes to each other and show the hierarchy of the subclasses of co-graphs. 


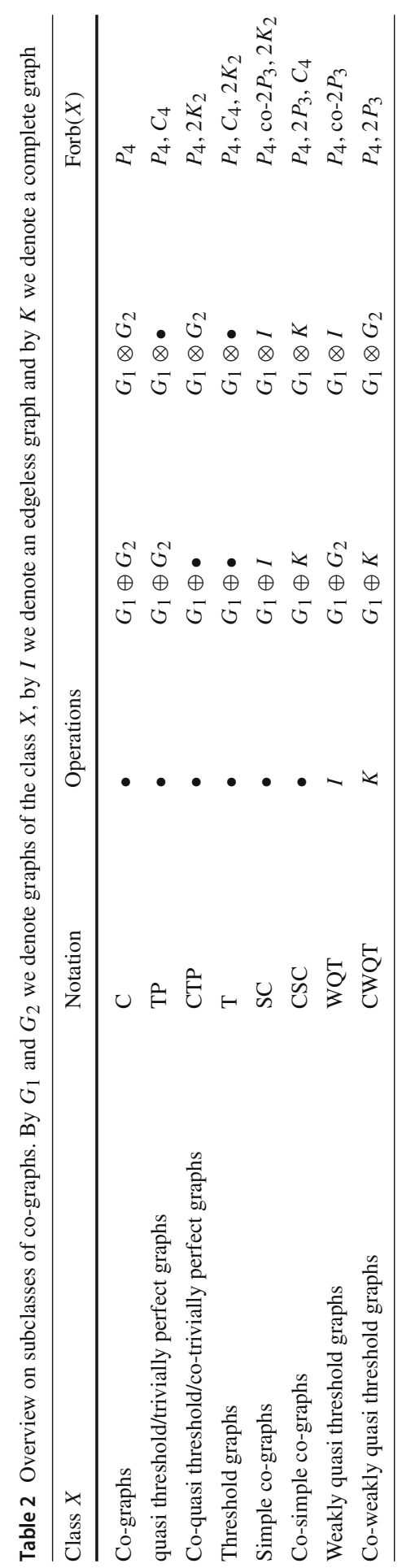


Fig. 1 Relations between the subclasses of co-graphs. If there is a path from $A$ to $B$, then it holds that $A \subset B$. The classes, that are not connected by a directed path are incomparable

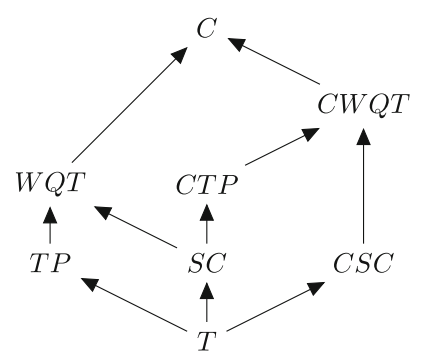

\section{Directed co-graphs and subclasses}

At first, we introduce operations that are used in the definition of directed co-graphs from Bechet et al. (1997) and show some interesting subclasses. Let $G_{1}=\left(V_{1}, E_{1}\right)$ and $G_{2}=\left(V_{2}, E_{2}\right)$ be two vertex-disjoint directed graphs. ${ }^{3}$

- The disjoint union of $G_{1}$ and $G_{2}$, denoted by $G_{1} \oplus G_{2}$, is the digraph with vertex set $V_{1} \cup V_{2}$ and arc set $E_{1} \cup E_{2}$.

- The series composition of $G_{1}$ and $G_{2}$, denoted by $G_{1} \otimes G_{2}$, is the digraph with vertex set $V_{1} \cup V_{2}$ and arc set $E_{1} \cup E_{2} \cup\left\{(u, v),(v, u) \mid u \in V_{1}, v \in V_{2}\right\}$.

- The order composition of $G_{1}$ and $G_{2}$, denoted by $G_{1} \oslash G_{2}$, is the digraph with vertex set $V_{1} \cup V_{2}$ and arc set $E_{1} \cup E_{2} \cup\left\{(u, v) \mid u \in V_{1}, v \in V_{2}\right\}$.

Every graph structure which can be obtained by this operations, can be constructed by a tree or even a sequence, as we could do for undirected co-graphs and threshold graphs. These trees/sequences can be used for algorithmic properties of those graphs.

\subsection{Directed co-graphs}

Definition 2 (Directed Co-Graphs Bechet et al. 1997) The class of directed co-graphs is recursively defined as follows.

(i) Every digraph on a single vertex $(\{v\}, \emptyset)$, denoted by $\bullet$, is a directed co-graph.

(ii) If $G_{1}$ and $G_{2}$ are directed co-graphs, then (a) $G_{1} \oplus G_{2}$, (b) $G_{1} \otimes G_{2}$, and (c) $G_{1} \oslash G_{2}$ are directed co-graphs.

The class of directed co-graphs is denoted by DC.

The recursive definition of directed and undirected co-graphs lead to the following observation.

Observation 3 For every directed co-graph $G$ the underlying undirected graph und $(G)$ is a co-graph.

The reverse direction only holds under certain conditions, see Theorem 4.

Obviously, for every directed co-graph we can define a tree structure, denoted as binary di-co-tree. While the leaves of the di-co-tree represent the vertices of the

\footnotetext{
3 We use the same symbols for the disjoint union and join between undirected and directed graphs. Although the meaning becomes clear from the context we want to emphasize this fact.
} 
Table 3 The eight forbidden induced subdigraphs for directed co-graphs

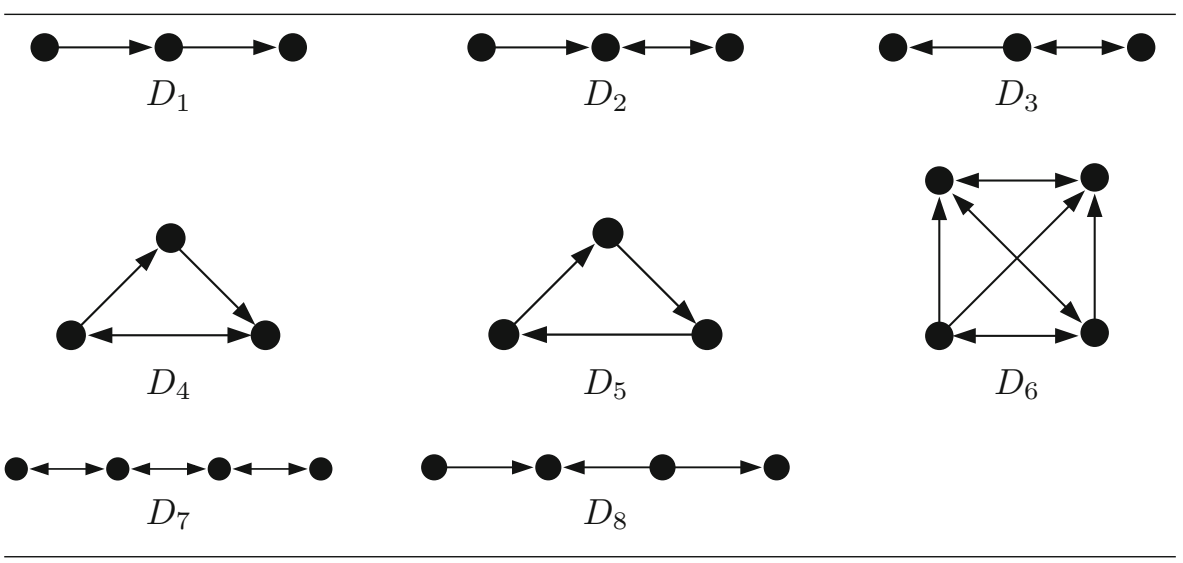

graph, the inner nodes of the di-co-tree correspond to the operations applied on the subexpressions which are defined by the associated subtrees. The construction of a binary di-co-tree for a directed co-graph is feasible in linear time (see Crespelle and Paul 2006).

From Bang-Jensen and Maddaloni (2014) we know that the weak $k$-linkage problem is solvable in polynomial time on directed co-graphs. The recursive structure leads to the existence of linear time dynamic programming algorithms for the computation of the size of a largest edgeless subdigraph, the size of a largest complete subdigraph, the size of a largest subdigraph that is a tournament and the size of a largest semicomplete subdigraph in directed co-graphs. Further, the hamiltonian path, hamiltonian cycle, regular subdigraph, and directed cut problem are computable in polynomial time on directed co-graphs (Gurski 2017). In addition, calculs of directed co-graphs are studied in connection with pomset logic in Retoré (1999). Recent results have also shown that directed path-width, directed tree-width, directed feedback vertex set number, cycle rank, DAG-depth and DAG-width are computable in linear time on directed co-graphs (Gurski and Rehs 2018b; Gurski et al. 2019b).

Lemma 2 (Gurski and Rehs 2018a) Let G be a digraph, then the following properties hold.

1. Digraph $G$ is a directed co-graph if and only if digraph co-G is a directed cograph.

2. Digraph $G$ is a directed co-graph if and only if digraph $G^{c}$ is a directed co-graph.

Moreover, the following properties hold for directed co-graphs:

Theorem 4 Let $G$ be a digraph. The following properties are equivalent:

1. $G$ is a directed co-graph.

2. $G \in$ Free $\left(\left\{D_{1}, \ldots, D_{8}\right\}\right)$ (see Table 3$)$.

3. $G \in$ Free $\left(\left\{D_{1}, \ldots, D_{6}\right\}\right)$ and und $(G) \in \operatorname{Free}\left(\left\{P_{4}\right\}\right)$.

4. $G \in$ Free $\left(\left\{D_{1}, \ldots, D_{6}\right\}\right)$ and und $(G)$ is a co-graph. 
Table 4 Forbidden induced subdigraphs for subclasses of directed co-graphs

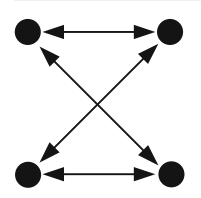

$D_{9}$

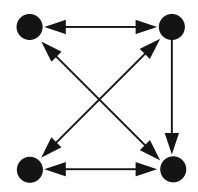

$D_{10}$

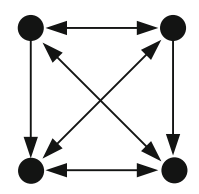

$D_{11}$

Table 5 Forbidden induced subdigraphs for subclasses of directed co-graphs

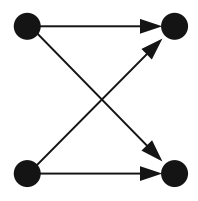

$D_{12}$

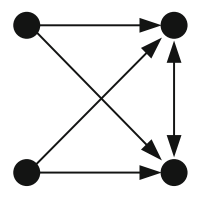

$D_{13}$

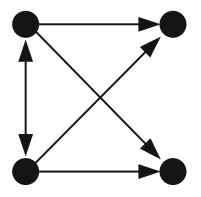

$D_{14}$

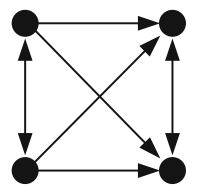

$D_{15}$

Table 6 Forbidden induced subdigraphs for subclasses of directed co-graphs

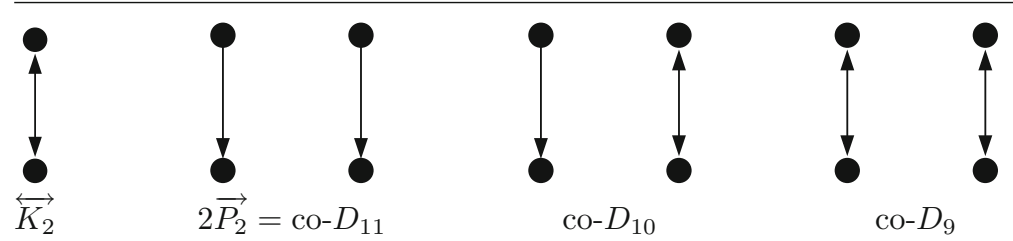

5. G has directed NLC-width $1 .^{4}$

6. $G$ has directed clique-width at most 2 and $G \in$ Free $\left(\left\{D_{2}, D_{3}\right\}\right){ }^{5}$

Proof (1) $\Leftrightarrow$ (2) By Crespelle and Paul (2006).

(3) $\Leftrightarrow$ (4) Since Forb $(\mathrm{C})=\left\{P_{4}\right\}$.

(1) $\Leftrightarrow$ (5) By Gurski et al. (2016).

(1) $\Leftrightarrow$ (6) By Gurski et al. (2016).

(2) $\Rightarrow$ (4) By Observation 3.

(3) $\Rightarrow$ (2) by Observation 2 .

For subclasses of directed co-graphs, which will be defined in the following subsections, some more forbidden subdigraphs are needed. Those are defined in Tables 4 , 5 , and 6 .

Observation 4 It holds:

1. $\left\{D_{1}, \ldots, D_{8}\right\}=\operatorname{co}-\left\{D_{1}, \ldots, D_{8}\right\}$.

\footnotetext{
4 For a definition of directed NLC-width (see Gurski et al. 2016).

5 For a definition of directed clique-width (see Courcelle and Olariu 2000).
} 
2. $\left\{D_{12}, \ldots, D_{15}\right\}=\operatorname{co}-\left\{D_{12}, \ldots, D_{15}\right\}$.

For directed co-graphs Observation 4 leads to the next result.

Proposition $1 D C=c o-D C$.

\subsection{Oriented co-graphs}

Beside directed co-graphs and their subclasses we also restrict these classes to oriented graphs by omitting the series operation.

Definition 3 (Oriented co-graphs) The class of oriented co-graphs is recursively defined as follows.

(i) A digraph on a single vertex $(\{v\}, \emptyset)$, denoted by $\bullet$, is an oriented co-graph.

(ii) If $G_{1}$ and $G_{2}$ are oriented co-graphs, then (a) $G_{1} \oplus G_{2}$ and (b) $G_{1} \oslash G_{2}$ are oriented co-graphs as well.

We denote the class of oriented co-graphs by OC.

The property of recursiveness of oriented and undirected co-graphs brings us to the following observation.

Observation 5 For every oriented co-graph $G$ the underlying undirected graph und $(G)$ is a co-graph.

The reverse direction holds only under special conditions, see Theorem 5. Oriented co-graphs were already studied by Lawler in Lawler (1976) and Corneil (1981, Section 5) where they are called transitive series parallel (TSP) digraphs. A digraph $G=$ $(V, A)$ is transitive if for every pair of $\operatorname{arcs}(u, v) \in A$ and $(v, w) \in A$ with $u \neq w$, it holds that arc $(u, w)$ is also part of $A$. For oriented co-graphs the oriented chromatic number and also the graph isomorphism problem can be solved in linear time (Gurski et al. 2019c, 2020c).

Lemma 3 Let $G$ be a digraph such that $G \in \operatorname{Free}\left(\left\{\overleftrightarrow{K_{2}}, D_{1}, D_{5}\right\}\right)$ then, $G$ is transitive.

Proof Let $(u, v),(v, w) \in A$ be two arcs of $G=(V, A)$. As $G \in \operatorname{Free}\left(\left\{\overleftrightarrow{K_{2}}\right\}\right)$ it also holds that $(v, u),(w, v) \notin A$. Additionally, since $G \in \operatorname{Free}\left(\left\{D_{1}, D_{5}\right\}\right)$ it holds that $(u, w) \in A$. This implies that $G$ is transitive.

Furthermore, the class OC can be defined by forbidden subdigraphs.

Theorem 5 (Gurski et al. 2019c) Let $G$ be a digraph. The following properties are equivalent:

1. $G$ is an oriented co-graph

2. $G \in$ Free $\left(\left\{D_{1}, D_{5}, D_{8}, \overleftrightarrow{K_{2}}\right\}\right)$.

3. $G \in \operatorname{Free}\left(\left\{D_{1}, D_{5}, \overleftrightarrow{K_{2}}\right\}\right)$ and und $(G) \in \operatorname{Free}\left(\left\{P_{4}\right\}\right)$.

4. $G \in$ Free $\left(\left\{D_{1}, D_{5}, \overleftrightarrow{K_{2}}\right\}\right)$ and und $(G)$ is a co-graph.

5. G has directed NLC-width 1 and $G \in$ Free $\left(\left\{\overleftrightarrow{K_{2}}\right\}\right)$. 
6. G has directed clique-width at most 2 and $G \in \operatorname{Free}\left(\left\{\overleftrightarrow{K_{2}}\right\}\right)$.

7. $G$ is transitive and $G \in \operatorname{Free}\left(\left\{\overleftrightarrow{K_{2}}, D_{8}\right\}\right)$.

Observation 6 Every oriented co-graph is a DAG.

By the results above, we can directly obtain the following known result.

Theorem 6 (Corneil et al. 1981) A graph $G$ is a co-graph if and only if there exists an orientation $G^{\prime}$ of $G$ such that $G^{\prime}$ is an oriented co-graph.

\subsection{Series-parallel partial order digraphs}

We take a look at the definitions of from Bang-Jensen and Gutin (2018) that base on Valdes et al. (1982). A series-parallel partial order is a partially ordered set $(X, \leq)$ that is constructed by the series composition and the parallel composition operation starting with a single element.

- Let $\left(X_{1}, \leq\right)$ and $\left(X_{2}, \leq\right)$ be two disjoint series-parallel partial orders, then distinct elements $x, y \in X_{1} \cup X_{2}$ of a series composition ${ }^{6}$ have the same order they have in $X_{1}$ or $X_{2}$. Respectively, this holds if both of them are from the same set, and $x \leq y$ if $x \in X_{1}$ and $y \in X_{2}$.

- Two elements $x, y \in X_{1} \cup X_{2}$ of a parallel composition are comparable if and only if both of them are in $X_{1}$ or both in $X_{2}$, while they keep their corresponding order.

Definition 4 (Series-parallel partial order digraphs) A series-parallel partial order digraph $G=(V, E)$ is a digraph, where $(V, \leq)$ is a series-parallel partial order and $(u, v) \in E$ if and only if $u \neq v$ and $u \leq v$.

We denote the class of series-parallel partial order digraphs by SPO.

For a digraph $G=(V, E)$ an edge $(u, v) \in E$ is symmetric if $(v, u) \in E$. Thus, each bidirectional arc is symmetric. Further, an edge is asymmetric if it is not symmetric, i.e. each edge with only one direction. We define the symmetric part of $G$ as $\operatorname{sym}(G)$, which is the spanning subdigraph of $G$ that contains exactly the symmetric arcs of $G$. Analogously, we define the asymmetric part of $G$ as $\operatorname{asym}(G)$, which is the spanning subdigraph with only asymmetric edges.

Moreover, Bechet et al. showed in Bechet et al. (1997) the following property of directed co-graphs.

Lemma 4 (Bechet et al. 1997) For every directed co-graph $G$ it holds that the asymmetric part of $G$ is a series-parallel partial order digraph and for the symmetric part the underlying undirected graph a co-graph.

The class of series-parallel partial ordered digraphs is equal to the class of oriented co-graphs since they have exactly the same recursive structure. Thus, this lemma is easy to prove with the following idea. Let $G$ be a directed co-graph and $T_{G}$ its corresponding di-co-tree.

\footnotetext{
${ }^{6}$ Note that the series composition in this case corresponds to the order composition in the definition of directed co-graphs.
} 
- Symmetric part Exchange each order composition with a directed union composition. Since there are no more oriented arcs left, this tree represents a co-graph.

- Asymmetric part Exchange each series composition with a directed union composition. Since there are no more bidirectional edges left, this tree represents an oriented co-graph, e.g. a series-parallel partial order digraph.

\subsection{Directed trivially perfect graphs}

Definition 5 (Directed trivially perfect graphs) The class of directed trivially perfect graphs is recursively defined as follows.

(i) Every digraph on a single vertex $(\{v\}, \emptyset)$, denoted by $\bullet$, is a directed trivially perfect graph.

(ii) If $G_{1}$ and $G_{2}$ are directed trivially perfect graphs, then $G_{1} \oplus G_{2}$ is a directed trivially perfect graph.

(iii) If $G$ is a directed trivially perfect graph, then (a) $G \oslash \bullet$, (b) $\bullet \oslash$, and (c) $G \otimes \bullet$ are directed trivially perfect graphs.

We denote the class of directed trivially perfect graphs by DTP.

The recursive definition of directed and undirected trivially perfect graphs lead to the following observation.

Observation 7 For every directed trivially perfect graph $G$ the underlying undirected graph und $(G)$ is a trivially perfect graph.

The reverse direction only holds under certain conditions, see Theorem 7.

Lemma 5 (Gurski et al. 2018) For every digraph $G$ the following statements are equivalent.

1. $G$ is a transitive tournament.

2. $G$ is an acyclic tournament.

3. $G \in$ Free $\left(\left\{\overrightarrow{C_{3}}\right\}\right)$ and $G$ is a tournament.

4. $G$ can be constructed from the one-vertex graph $K_{1}$ by repeatedly adding an out-dominating vertex.

5. $G$ can be constructed from the one-vertex graph $K_{1}$ by repeatedly adding an in-dominated vertex.

6. $G \in \operatorname{Free}\left(\left\{2 \overleftrightarrow{K_{1}}, \overleftrightarrow{K_{2}}, D_{5}\right\}\right)$.

7. $G$ is transitive and $G \in$ Free $\left(\left\{2 \overleftrightarrow{K_{1}}, \overleftrightarrow{K_{2}}\right\}\right)$.

The class DTP can also be defined by forbidden induced subdigraphs as the following theorem shows.

Theorem 7 Let $G$ be a digraph. The following properties are equivalent:

1. G is a directed trivially perfect graph.

2. $G \in$ Free $\left(\left\{D_{1}, \ldots, D_{15}\right\}\right)$.

3. $G \in$ Free $\left(\left\{D_{1}, D_{2}, D_{3}, D_{4}, D_{5}, D_{6}, D_{10}, D_{11}, D_{13}, D_{14}, D_{15}\right\}\right)$ and und $(G) \in$ Free $\left(\left\{C_{4}, P_{4}\right\}\right)$. 
4. $G \in$ Free $\left(\left\{D_{1}, D_{2}, D_{3}, D_{4}, D_{5}, D_{6}, D_{10}, D_{11}, D_{13}, D_{14}, D_{15}\right\}\right)$ and und $(G)$ is a trivially perfect graph.

Proof $(1) \Rightarrow(2)$ Holds since the given forbidden digraphs $D_{1}, \ldots, D_{15} \notin$ DTP and the set of directed trivially perfect graphs is closed under taking induced subdigraphs.

(2) $\Rightarrow$ (1) Since $G \in \operatorname{Free}\left(\left\{D_{1}, D_{2}, D_{3}, D_{4}, D_{5}, D_{6}, D_{7}, D_{8}\right\}\right)$ digraph $G$ is a directed co-graph by Crespelle and Paul (2006) and consequently, there exists a construction using disjoint union, series composition, and order composition.

As $G \in \operatorname{Free}\left(\left\{D_{9}, D_{10}, D_{11}\right\}\right)$ it holds that for every series composition between two graphs with at least two vertices at least one of the graphs has to be bidirectional complete. Such a subdigraph can be inserted by a number of operations that are allowed in directed trivially perfect graphs.

As $G \in \operatorname{Free}\left(\left\{D_{12}, D_{13}, D_{14}, D_{15}\right\}\right)$ holds, we know that for every order combination between two graphs on at least two vertices, at least one of the graphs must be a tournament. As $G \in \operatorname{Free}\left(\left\{D_{5}\right\}\right)=\operatorname{Free}\left(\left\{C_{3}\right\}\right)$ by Lemma 5 we even can say that at least one of the graphs is a transitive tournament. A digraph like this can be defined by a sequence of outdominating or indominating vertices (Lemma 5) which are also feasible operations for directed trivially perfect graphs.

(3) $\Leftrightarrow$ (4) As Forb (TP) $=\left\{C_{4}, P_{4}\right\}$.

(2) $\Rightarrow$ (4) By Observation 7.

(3) $\Rightarrow$ (2) By Observation 2.

For directed trivially perfect graphs Observation 4 with Table 4 and Table 6 lead to the next result.

\section{Proposition $2 D T P \neq c o-D T P$}

This motivates us to consider the class of edge complements of directed trivially perfect graphs.

Definition 6 (Directed co-trivially perfect graphs) The class of directed co-trivially perfect graphs is recursively defined as follows.

(i) Every digraph on a single vertex $(\{v\}, \emptyset)$, denoted by $\bullet$, is a directed co-trivially perfect graph.

(ii) If $G_{1}$ and $G_{2}$ are directed co-trivially perfect graphs, then $G_{1} \otimes G_{2}$ is a directed trivially perfect graph.

(iii) If $G$ is a directed co-trivially perfect graph, then (a) $G \oslash \bullet$, (b) $\bullet \oslash G$, and (c) $G \oplus \bullet$ are directed co-trivially perfect graphs.

We denote the class of directed co-trivially perfect graphs by DCTP.

Theorem 7 and Lemma 1 lead to the following characterization for directed cotrivially perfect graphs.

Theorem 8 Let $G$ be a digraph. The following properties are equivalent:

1. $G$ is a directed co-trivially perfect graph.

2. $G \in$ Free $\left(\left\{D_{1}, \ldots, D_{8}\right.\right.$, co- $D_{9}$, co- $D_{10}$, co- $\left.\left.D_{11}, D_{12}, \ldots, D_{15}\right\}\right)$.

3. $G \in$ Free $\left(\left\{D_{1}, \ldots, D_{6}, D_{12}, \ldots, D_{15}\right\}\right)$ and und $(G) \in$ Free $\left(\left\{P_{4}, 2 K_{2}\right\}\right)$.

4. $G \in$ Free $\left(\left\{D_{1}, \ldots, D_{6}, D_{12}, \ldots, D_{15}\right\}\right)$ and und $(G)$ is a co-trivially perfect graph. 


\subsection{Oriented trivially perfect graphs}

Definition 7 (Oriented trivially perfect graphs) The class of oriented trivially perfect graphs is recursively defined as follows.

(i) Every digraph on a single vertex $(\{v\}, \emptyset)$, denoted by $\bullet$, is an oriented trivially perfect graph.

(ii) If $G_{1}$ and $G_{2}$ are oriented trivially perfect graphs, then $G_{1} \oplus G_{2}$ is an oriented trivially perfect graph.

(iiii) If $G$ is an oriented trivially perfect graph, then (a) $G \oslash \bullet$ and (b) $\bullet \oslash G$ are oriented trivially perfect graphs.

We denote the class of oriented trivially perfect graphs by OTP.

The recursive definition of oriented and undirected trivially perfect graphs lead to the following observation.

Observation 8 For every oriented trivially perfect graph $G$ the underlying undirected graph und $(G)$ is a trivially perfect graph.

Similar as for oriented co-graphs we obtain a definition of OTP by forbidden induced subdigraphs.

Theorem 9 Let $G$ be a digraph. The following properties are equivalent:

1. $G$ is an oriented trivially perfect graph.

2. $G \in$ Free $\left(\left\{D_{1}, D_{5}, D_{8}, D_{12}, \overleftrightarrow{K_{2}}\right\}\right)$

3. $G \in$ Free $\left(\left\{D_{1}, D_{5}, \overleftrightarrow{K_{2}}\right\}\right)$ and und $(G) \in$ Free $\left(\left\{C_{4}, P_{4}\right\}\right)$.

4. $G \in \operatorname{Free}\left(\left\{D_{1}, D_{5}, \overleftrightarrow{K_{2}}\right\}\right)$ and und $(G)$ is a trivially perfect graph.

5. $G$ is transitive and $G \in$ Free $\left(\left\{\overleftrightarrow{K_{2}}, D_{8}, D_{12}\right\}\right)$

Proof $(1) \Rightarrow(2)$ If $G$ is an oriented trivially perfect graph, then $G$ is a directed trivially perfect graph and $G \in$ Free $\left(\left\{D_{1}, \ldots, D_{15}\right\}\right)$. Further $G \in$ Free $\left(\left\{\overleftrightarrow{K_{2}}\right\}\right)$ because of the missing series composition. This leads to $G \in \operatorname{Free}\left(\left\{D_{1}, D_{5}, D_{8}, D_{12}, \overleftrightarrow{K_{2}}\right\}\right)$.

(2) $\Rightarrow$ (1) If $G \in \operatorname{Free}\left(\left\{D_{1}, D_{5}, D_{8}, D_{12}, \overleftrightarrow{K_{2}}\right\}\right)$, then $G \in \operatorname{Free}\left(\left\{D_{1}, \ldots, D_{15}\right\}\right)$ and is a directed trivially perfect graph. Since $G \in \operatorname{Free}\left(\left\{\overleftrightarrow{K_{2}}\right\}\right)$ there is no series operation in any construction of $G$ which implies that $G$ is an oriented trivially perfect graph.

(3) $\Leftrightarrow$ (4) Since Forb(TP) $=\left\{C_{4}, P_{4}\right\}$.

(2) $\Rightarrow$ (4) By Observation 8 .

(3) $\Rightarrow$ (2) By Observation 2 .

(2) $\Rightarrow(5)$ By Lemma 3 we know that $G$ is transitive.

$(5) \Rightarrow$ (2) If $G$ is transitive, then $G$ has no induced $D_{1}, D_{5}$.

Theorem 10 A graph $G$ is a trivially perfect graph if and only if there exists an orientation $G^{\prime}$ of $G$ such that $G^{\prime}$ is an oriented trivially perfect graph.

Proof Let $G$ be a trivially perfect graph. Then $G$ is also a comparability graph (see Golumbic 1980), which implies that $G$ has a transitive orientation $G^{\prime}$. Since $G \in$ 
Free $\left(\left\{C_{4}, P_{4}\right\}\right)$ it follows that $G^{\prime} \in \operatorname{Free}\left(\left\{D_{8}, D_{12}\right\}\right)$. Further by definition $G^{\prime} \in$ Free $\left(\left\{\overleftrightarrow{K_{2}}\right\}\right)$. By Theorem 9 we know that $G^{\prime}$ is an oriented trivially perfect graph.

For the reverse direction let $G^{\prime}$ be an oriented trivially perfect graph. Then by Theorem 9 it holds that $G^{\prime} \in \operatorname{Free}\left(\left\{D_{8}, D_{12}\right\}\right)$. Since $D_{8}$ is the only transitive orientation of $P_{4}$ and $D_{12}$ is the only transitive orientation of $C_{4}$ it holds that und $(G) \in \operatorname{Free}\left(\left\{C_{4}, P_{4}\right\}\right)$. Thus, und $(G)$ is a trivially perfect graph.

Observation 9 If $G \in D T P$, then the underlying undirected graph of the symmetric part of $G$ is trivially perfect and the asymmetric part of $G$ is an oriented trivially perfect digraph.

This holds since the asymmetric part is constructed according to exactly the same rules as trivially perfect graphs and the asymmetric part is constructed according to the rules of OTP.

Definition 8 (Oriented co-trivially perfect graphs) The class of oriented co-trivially perfect graphs is recursively defined as follows.

(i) Every digraph on a single vertex $(\{v\}, \emptyset)$, denoted by $\bullet$, is an oriented co-trivially perfect graph.

(ii) If $G$ is an oriented co-trivially perfect graph, then (a) $G \oslash \bullet$, (b) $\bullet \oslash G$, and (c) $G \oplus \bullet$ are oriented co-trivially perfect graphs.

We denote the class of oriented co-trivially perfect graphs by OCTP.

Restricting the operations of directed co-trivially perfect graphs to oriented graphs leads to the same operations as the class of orientated threshold graphs, which will be considered in Sect. 4.15.

\subsection{Directed weakly quasi threshold graphs}

Definition 9 (Directed weakly quasi threshold graphs) The class of directed weakly quasi threshold graphs is recursively defined as follows.

(i) Every edgeless digraph is a directed weakly quasi threshold graph.

(ii) If $G_{1}$ and $G_{2}$ are directed weakly quasi threshold graphs, then $G_{1} \oplus G_{2}$ is a directed weakly quasi threshold graph.

(iii) If $G$ is a directed weakly quasi threshold graph and $I$ is an edgeless digraph, then (a) $G \oslash I$, (b) $I \oslash G$, and (c) $G \otimes I$ are directed weakly quasi threshold graphs.

We denote the class of directed weakly quasi threshold graphs by DWQT.

Observation 10 If $G$ is a directed weakly quasi threshold graph, und $(G)$ is weakly quasi threshold graph.

Theorem 11 Let $G$ be a digraph. The following properties are equivalent:

1. G is a directed weakly quasi threshold graph.

2. $G \in \operatorname{Free}\left(\left\{D_{1}, \ldots, D_{8}, Q_{1}, \ldots, Q_{7}\right\}\right)^{7}$.

\footnotetext{
$\overline{7 \text { Note that } Q_{1}=D_{11} \text { and } Q_{2}}=D_{15}$.
} 
Table 7 Digraphs for the construction of forbidden subdigraphs of DWQT

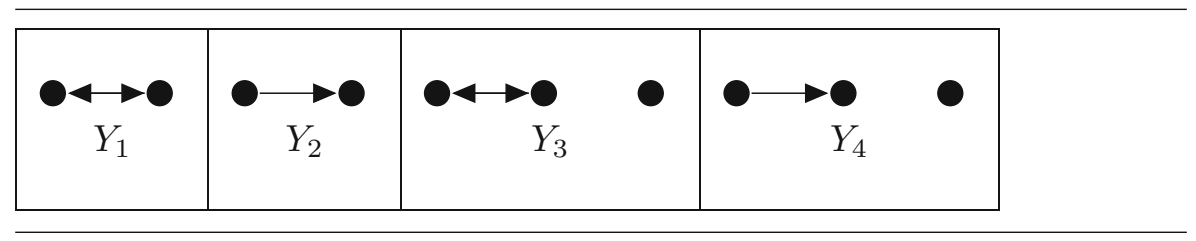

Table 8 Constructions of the forbidden subdigraphs of DWQT

\begin{tabular}{llll}
\hline$Q_{1}=Y_{2} \otimes Y_{2}$ & $Q_{2}=Y_{1} \oslash Y_{1}$ & $Q_{3}=Y_{3} \otimes Y_{3}$ & $Q_{4}=Y_{2} \otimes Y_{3}$ \\
$Q_{5}=Y_{1} \oslash Y_{4}$ & $Q_{6}=Y_{4} \oslash Y_{1}$ & $Q_{7}=Y_{4} \oslash Y_{4}$ & \\
\hline
\end{tabular}

3. $G \in$ Free $\left(\left\{D_{1}, \ldots, D_{6}, Q_{1}, Q_{2}, Q_{4}, Q_{5}, Q_{6}\right\}\right)$ and und $(G) \in$ Free $\left(\left\{P_{4}\right.\right.$, co- $\left.\left.2 P_{3}\right\}\right)$.

4. $G \in$ Free $\left(\left\{D_{1}, \ldots, D_{6}, Q_{1}, Q_{2}, Q_{4}, Q_{5}, Q_{6}\right\}\right)$ and und $(G)$ is a weakly quasi threshold graph.

Proof (1) $\Rightarrow$ (2) The forbidden digraphs $D_{1}, \ldots, D_{8}, Q_{1}, \ldots, Q_{7}$ are not directed weakly quasi threshold graphs and the set of directed weakly quasi threshold graphs is hereditary.

(2) $\Rightarrow$ (1) Let $G$ be a digraph without induced $D_{1}, \ldots, D_{8}, Q_{1}, \ldots, Q_{7}$. As there are no induced $D_{1}, \ldots, D_{8}$, it holds that $G \in \mathrm{DC}$. Thus, $G$ is constructed by the disjoint union, the series and the order composition. $Q_{1}, Q_{3}$ and $Q_{4}$ can only be constructed by a series composition of two graphs $G_{1}$ and $G_{2}$, where $G_{1}, G_{2} \in\left\{Y_{2}, Y_{3}\right\}$, see Tables 7 and 8 . We underline that $Y_{2}, Y_{3}$ are contained in every directed co-graph containing more vertices, which is not a sequence of length at least one of series compositions of independent sets. Thus, there are no bigger forbidden induced subdigraphs that are constructed by a series operation, with the consequence that the $Q_{1}, Q_{3}$ and $Q_{4}$ characterize exactly the allowed series compositions in DWQT.

The only way to construct $Q_{2}, Q_{5}, Q_{6}$ and $Q_{7}$ is by an order composition of two graphs $G_{1}$ and $G_{2}$, where $G_{1}, G_{2} \in\left\{Y_{1}, Y_{4}\right\}$. We note that $Y_{1}, Y_{4}$ are contained in every directed co-graph containing more vertices, that is not a sequence of length at least one of order compositions of independent sets. Hence, there are no forbidden induced subdigraphs containing more vertices that are constructed by an order operation, such that the $Q_{2}, Q_{5}, Q_{6}$ and $Q_{7}$ characterize exactly the allowed order compositions in DWQT. Finally, by excluding these digraphs, we end up in the Definition 9 for DWQT, such that $G \in$ DWQT.

(2) $\Rightarrow$ (4) By Observation 10.

(3) $\Rightarrow$ (2) By Observation 2.

(3) $\Leftrightarrow(4)$ Since Forb (WQT) $=\left\{P_{4}, \operatorname{co}-2 P_{3}\right\}$.

Since co- $\left\{Q_{1}, \ldots, Q_{7}\right\} \neq\left\{Q_{1}, \ldots, Q_{7}\right\}$ holds, it is reasonable to introduce the complementary class of DWQT. 
Table 9 Forbidden induced subdigraphs for DWQT

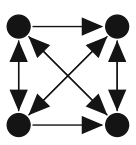

$Q_{1}$

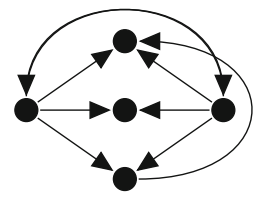

$Q_{5}$

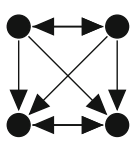

$Q_{2}$

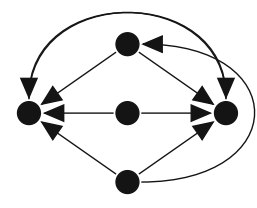

$Q_{6}$

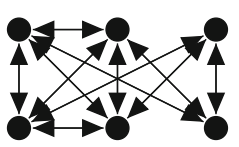

$Q_{3}$

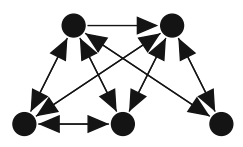

$Q_{4}$

\subsection{Oriented weakly quasi threshold graphs}

Definition 10 (Oriented weakly quasi threshold graphs) The class of oriented weakly quasi threshold graphs is recursively defined as follows.

(i) Every edgeless digraph is an oriented weakly quasi threshold graph.

(ii) If $G_{1}$ and $G_{2}$ are oriented weakly quasi threshold graphs, then $G_{1} \oplus G_{2}$ is an oriented weakly quasi threshold graph.

(iii) If $G$ is an oriented weakly quasi threshold graph and $I$ is an edgeless digraph, then (a) $G \oslash I$ and (b) $I \oslash G$ are oriented weakly quasi threshold graphs.

We denote the class of oriented weakly quasi threshold graphs by OWQT.

Theorem 12 Let $G$ be a digraph. The following properties are equivalent:

1. G is a oriented weakly quasi threshold graph.

2. $G \in$ Free $\left(\left\{D_{1}, D_{5}, D_{8}, \overleftrightarrow{K_{2}}, Q_{7}\right\}\right)$.

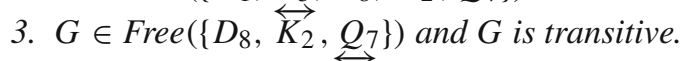

4. $G \in$ Free $\left(\left\{D_{1}, D_{5}, \overleftrightarrow{K_{2}}\right\}\right)$ and und $(G)$ is a weakly quasi threshold graph.

Proof $(1) \Rightarrow(2)$ Let $G$ be in OWQT. Since OWQT $\subset$ OC we know that $D_{1}, D_{5}, D_{8}$ and $\overleftrightarrow{K_{2}}$ are forbidden. Further, since OWQT $\subset$ DWQT the graphs of Table 9 are forbidden. As $\overleftrightarrow{K_{2}}$ is forbidden as well, this reduces the table and only $Q_{7}$ remains. Since OWQT is closed under taking induced subdigraphs, this holds for every digraph in this class.

$(2) \Rightarrow$ (1) Since $D_{1}, D_{5}, D_{8}$ and $\overleftrightarrow{K_{2}}$ are forbidden induced subdigraphs of $G$ it holds that $G$ is an oriented co-graph, see Theorem 5. Consequently, $G$ has been constructed by the operations allowed for oriented co-graphs, which are the disjoint union and the order composition. Further, $Q_{7}$ is excluded since it must have been the result of an order composition. Since $Q_{7}=Y_{4} \oslash Y_{4}$ and $Y_{4}$ is neither an edgeless digraph nor a transitive tournament, this order composition is not valid in OWQT and thus, $Q_{7}$ is a forbidden subdigraph. For every other possible order composition of two 
oriented digraphs $G_{1}$ and $G_{2}$, either $G_{1}$ or $G_{2}$ is edgeless or a transitive tournament, which is allowed in OWQT, or both graphs contain $Y_{4}$ as induced subdigraph, such that the forbidden $Q_{7}$ completely characterizes the restriction of the order compositions in OWQT. Since $G$ does not contain one of these forbidden subdigraphs, $G \in$ OWQT follows.

(2) $\Rightarrow$ (3) Follows by Lemma 5 .

(3) $\Rightarrow(2)$ The transitivity of $G$ implies that $D_{1}$ and $D_{5}$ are forbidden, as they do not satisfy the definition of transitivity.

$(2) \Leftrightarrow(4)$ Since Forb $($ WQT $)=\left\{P_{4}\right.$, co- $\left.2 P_{3}\right\}$.

Observation 11 If $G \in D W Q T$, then the underlying undirected graph of the symmetric part of $G$ is a weakly quasi threshold graph and the asymmetric part of $G$ is an oriented weakly quasi threshold graph.

This holds since the asymmetric part is constructed with the same rules as weakly quasi threshold graphs and the asymmetric part with the rules of OWQT.

\subsection{Directed co-weakly quasi threshold graphs}

Definition 11 (Directed co-weakly quasi threshold graphs) The class of directed coweakly quasi threshold graphs is recursively defined as follows.

(i) Every bidirectional complete digraph is a directed co-weakly quasi threshold graph.

(ii) If $G_{1}$ and $G_{2}$ are directed co-weakly quasi threshold graphs, then $G_{1} \otimes G_{2}$ is a directed co-weakly quasi threshold graph.

(iii) If $G$ is a directed co-weakly quasi threshold graph and $K$ is a bidirectional complete digraph, then (a) $G \oslash K$, (b) $K \oslash G$, and (c) $G \oplus K$ are directed co-weakly quasi threshold graphs.

We denote the class of directed co-weakly quasi threshold graphs by DCWQT.

Theorem 13 For a graph $G$ the following properties are equivalent.

1. $G \in D C W Q T$.

2. $G \in$ Free $\left(\left\{D_{1}, \ldots, D_{8}\right.\right.$, co- $Q_{1}, \ldots$, co- $\left.\left.Q_{7}\right\}\right)$, see Table 10 .

Proof By Lemma 1, we know that co-DWQT $=$ Free $\left(\operatorname{co}-\left\{D_{1}, \ldots, D_{8}, Q_{1}, \ldots, Q_{7}\right\}\right)$. Since DCWQT $=$ co-DWQT, it follows by Theorem 11 that the forbidden induced subdigraphs are $D_{1}, \ldots, D_{8}$ since they are self complementary, and co- $Q_{1}, \ldots$, co- $Q_{7}$.

\subsection{Oriented co-weakly quasi threshold graphs}

Definition 12 (Oriented co-weakly quasi threshold graphs) The class of oriented coweakly quasi threshold graphs is recursively defined as follows.

(i) Every transitive tournament is an oriented co-weakly quasi threshold graph. 
Table 10 Forbidden induced subdigraphs for DCWQT

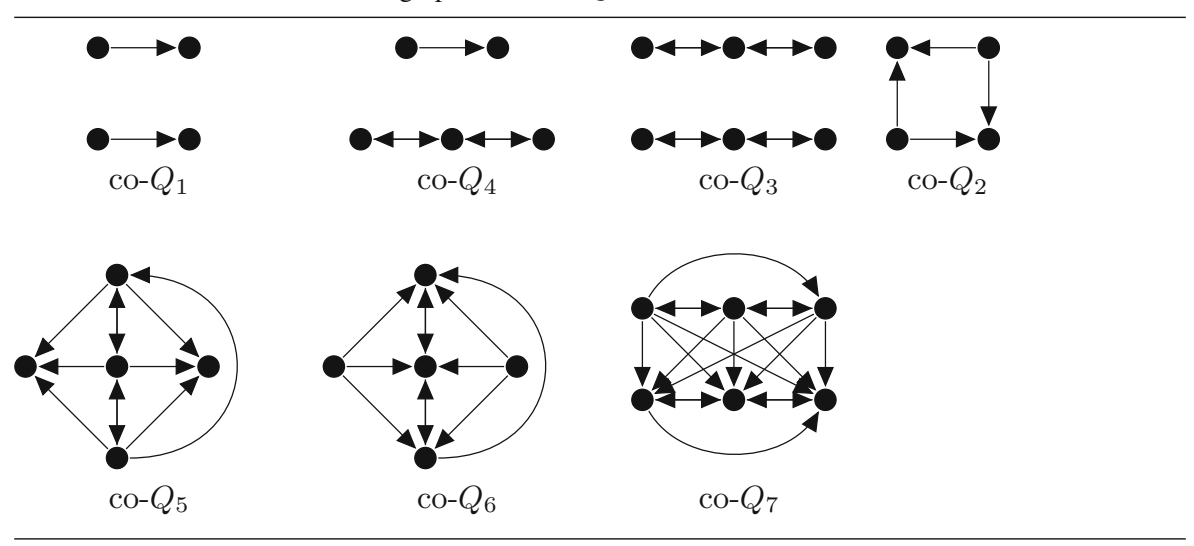

Table 11 Forbidden induced subdigraphs of OCWQT

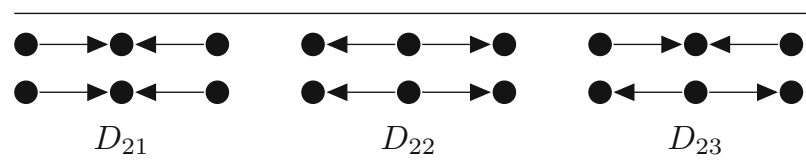

Table 12 Digraphs for the construction of forbidden subdigraphs of OCWQT

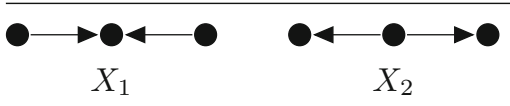

(ii) If $G$ is an oriented co-weakly quasi threshold graph and $T$ is a transitive tournament, then (a) $G \oslash T$, (b) $T \oslash G$, and (c) $G \oplus T$ are oriented co-weakly quasi threshold graphs.

We denote the class of oriented co-weakly quasi threshold graphs by OCWQT.

Obviously, a transitive tournament $\vec{T}_{n}$ is a subdigraph, but not an induced subdigraph of $\overleftrightarrow{K_{n}}$. Thus, the operations allowed in OCWQT are not exactly building the complement of the digraphs in DCWQT, but since biorientations are forbidden in oriented digraphs, it is useful to define the class as above.

Theorem 14 For a graph $G$ the following properties are equivalent.

1. $G$ is a oriented co-weakly quasi threshold graph.

2. $G \in$ Free $\left(\left\{D_{1}, D_{5}, D_{8}, \overleftrightarrow{K_{2}}, D_{12}, D_{21}, D_{22}, D_{23}\right\}\right)^{8}$.

3. $G$ is an oriented co-graph and $G \in$ Free $\left(\left\{D_{12}, D_{21}, D_{22}, D_{23}\right\}\right)$.

4. $G \in$ Free $\left(\left\{D_{8}, \overleftrightarrow{K_{2}}, D_{12}, D_{21}, D_{22}, D_{23}\right\}\right)$ and $G$ is transitive.

$8 D_{12}$ is equal to co- $Q_{2}$. 
Proof $(1) \Rightarrow$ (2) Let $G \in$ OCWQT and assume $G$ contains one of $D_{1}, D_{5}, D_{8}, \overleftrightarrow{K_{2}}$ $D_{12}, D_{21}, D_{22}, D_{23}$. Since these digraphs are not constructed by the operations of OCWQT, this contradicts that $G$ is in this class. Since OCWQT is closed under taking induced subdigraphs, $G$ cannot contain one of these graphs as induced subdigraphs.

$(2) \Rightarrow(1)$ Since $D_{1}, D_{5}, D_{8}$ and $\overleftrightarrow{K_{2}}$ are forbidden induced subdigraphs of $G$, $G$ is an oriented co-graph, see Theorem 14 . Thus, $G$ has been constructed by the operations allowed for oriented co-graphs, which are the disjoint union and the order composition.

Since $D_{12}$ is not an induced subdigraph of $G$, in every order composition of two graphs $G_{1}$ and $G_{2}$ in the construction of $G$, at least one of them must have been a transitive tournament. For every bigger digraph, composed by an order composition of two oriented digraphs $G_{1}$ and $G_{2}$, it holds that either one of them is a transitive tournament, which is allowed in the order composition in OCWQT, or else $I_{2}$ is included as induced subdigraph in $G_{1}$ and $G_{2}$, which means that $D_{12}$ is contained as induced subdigraph. This leads to the conclusion that prohibiting $D_{12}$ exactly characterizes the order composition allowed in OCWQT.

Since $D_{21}, D_{22}$ and $D_{23}$ are not connected, they must be the result of the disjoint union of two oriented co-graphs $G_{1}$ and $G_{2}$, with $G_{1}, G_{2} \in\left\{X_{1}, X_{2}\right\}$, see Tables 11 and 12. As $X_{1}$ and $X_{2}$ are neither transitive tournaments nor edgeless digraphs, they are forbidden in OCWQT. Every bigger oriented digraph emerging by a disjoint union either contains $D_{21}, D_{22}$ and $D_{23}$, or is feasible to construct by adding transitive tournaments one by one. Consequently, excluding $D_{21}, D_{22}$ and $D_{23}$ exactly characterizes the restriction of the disjoint union in OCWQT.

This exactly leads to Definition 12 of OWQT, such that $G \in$ OCWQT.

(2) $\Leftrightarrow$ (3) Holds by Theorem 5 .

(2) $\Rightarrow$ (4) Holds by Lemma 5 .

$(4) \Rightarrow(2)$ The transitivity of $G$ implies that there cannot be $D_{1}$ or $D_{5}$, as both do not satisfy the definition of transitivity.

The next class we consider is the class of simple co-graphs, of which we construct a directed version.

\subsection{Directed simple co-graphs}

Definition 13 (Directed simple co-graphs) The class of directed simple co-graphs is recursively defined as follows.

(i) Every digraph on a single vertex $(\{v\}, \emptyset)$, denoted by $\bullet$, is a directed simple cograph.

(ii) If $G$ is a directed simple co-graph $I$ is an edgeless digraph, then (a) $G \oplus I$, (b) $G \oslash I$, (c) $I \oslash G$, and (d) $G \otimes I$ are directed simple co-graphs.

We denote the class of directed simple co-graphs by DSC.

Observation 12 If $G$ is a directed simple co-graph, und $(G)$ is a simple co-graph.

Since directed simple co-graphs are a subset of directed weakly quasi threshold graphs, they can be defined by adding further subdigraphs to those given for 
directed weakly quasi threshold graphs. These have to ensure that for every disjoint union of $G_{1}$ and $G_{2}$ either $G_{1}$ or $G_{2}$ has no edges. This can be done by excluding co- $D_{11}$, co- $D_{10}$, co- $D_{9}$, see Table 13 .

Theorem 15 The following statements are equivalent.

1. $G \in D S C$.

2. $G \in$ Free $\left(\left\{D_{1}, \ldots, D_{8}, Q_{1}, \ldots, Q_{7}\right.\right.$, co- $D_{9}$, co- $D_{10}$, co- $\left.\left.D_{11}\right\}\right)$.

3. $G \in$ Free $\left(\left\{D_{1}, \ldots, D_{8}, Q_{1}, \ldots, Q_{7}\right\}\right)$ and und $(G) \in$ Free $\left(\left\{P_{4}\right.\right.$, co- $\left.\left.2 P_{3}, 2 K_{2}\right\}\right)$.

4. $G \in$ Free $\left(\left\{D_{1}, \ldots, D_{8}, Q_{1}, \ldots, Q_{7}\right\}\right)$ and und $(G) \in S C$.

Proof $(1) \Rightarrow$ (2) The given forbidden digraphs $D_{1}, \ldots, D_{8}, Q_{1}, \ldots, Q_{7}$, co- $D_{9}$, co- $D_{10}$ and co- $D_{11}$ are no directed simple co-graphs and the set of directed directed simple co-graphs is hereditary.

$(2) \Rightarrow$ (1) Let $G \in \operatorname{Free}\left(\left\{D_{1}, \ldots, D_{8}, Q_{1}, \ldots, Q_{7}\right.\right.$, co- $D_{9}$, co- $D_{10}$, co- $\left.\left.D_{11}\right\}\right)$. Then, we know by Theorem 11 that $G$ is in DWQT, such that in every series and order composition of two digraphs $G_{1}$ and $G_{2}$, either $G_{1}$ or $G_{2}$ must be an edgeless digraph (see proof of Theorem 11), exactly as in the definition of DSC. By excluding co- $D_{9}$, co- $D_{10}$ and co- $D_{11}$ there is no disjoint union $G_{1} \oplus G_{2}$ allowed, in which at least one of the two digraphs contains an edge. Therefore, one of $G_{1}$ or $G_{2}$ must be edgeless, which exactly is the restriction for the disjoint union from Definition 13. For every other digraph built by $G_{1} \oplus G_{2}$, either $G_{1}$ and $G_{1}$ contain edges, such that co- $D_{9}$, co- $D_{10}$ or co- $D_{11}$ is an induced subdigraph, or $G_{1}$ or $G_{1}$ contains no edges, which leads to a legit digraph of DSC. Finally, we end up in Definition 13. Consequently, $G$ must be in DSC.

(2) $\Rightarrow$ (4) By Observation 12 .

(3) $\Rightarrow$ (2) By Observation 2.

(3) $\Leftrightarrow$ (4) Since Forb $(\mathrm{SC})=\left\{P_{4}\right.$, co- $\left.2 P_{3}, 2 K_{2}\right\}$.

\subsection{Oriented simple co-graphs}

Definition 14 (Oriented simple co-graphs) The class of oriented simple co-graphs is recursively defined as follows.

(i) Every digraph on a single vertex $(\{v\}, \emptyset)$, denoted by $\bullet$, is an oriented simple co-graph.

(ii) If $G$ is an oriented simple co-graph $I$ is an edgeless digraph, then (a) $G \oplus I$, (b) $G \oslash I$, (c) $I \oslash G$ are oriented simple co-graphs.

We denote the class of oriented simple co-graphs by OSC.

Theorem 16 Let $G$ be a digraph. The following properties are equivalent:

1. $G$ is an oriented simple co-graph.

2. $G \in$ Free $\left(\left\{D_{1}, D_{5}, D_{8}, Q_{7}\right.\right.$, co- $\left.\left.D_{11}, \overleftrightarrow{K_{2}}\right\}\right)$.

3. $G \in$ Free $\left(\left\{D_{8}, Q_{7}\right.\right.$, co- $\left.\left.D_{11}, \overleftrightarrow{K_{2}}\right\}\right)$ and $G$ is transitive. 
Table 13 Complementary graphs of $D_{9}, D_{10}$ and $D_{11}$

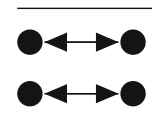

co- $D_{9}$

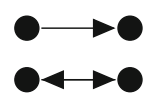

co- $D_{10}$

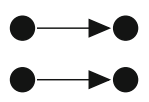

co- $D_{11}$

Proof (1) $\Rightarrow$ (2) Let $G$ be in OSC. Since OSC $\subset$ DSC and OSC $\subset$ OC we know that the forbidden induced subdigraphs of DSC, as well as the forbidden induced subdigraphs of OC, must also be excluded in OSC. Consequently, none of the graphs $D_{1}, D_{5}, D_{8}, Q_{7}$, co- $D_{11}, \overleftrightarrow{K_{2}}$ can be induced subdigraphs of $G$. Since the class OSC is closed under taking induced subdigraphs this holds for every digraph of the class.

$(2) \Rightarrow(1)$ Let $G$ contain none of the digraphs from above as induced subdigraph.

Since $G$ has no induced $D_{1}, D_{5}, D_{8}$ and $\overleftrightarrow{K_{2}}, G$ must be an oriented co-graph by Theorem 5 and thus, $G$ must have been built by a disjoint union or a series composition. Further, we know from the proof of Theorem 15 which forbidden subdigraphs are exactly leading to the restriction of the disjoint union and the order composition. Since $G$ has no induced $\overleftrightarrow{K_{2}}$, the list reduces to $Q_{7}$ and co- $D_{11}$. With the same argumentation as in the proof of Theorem 15, every other digraph that is constructed by a directed union or an order composition is either legit for this class, or contains one of these forbidden digraphs as subdigraph. Consequently, this leads exactly to the definition of OSC, such that $G$ must be in OSC.

(2) $\Rightarrow$ (3) Holds with Lemma 5 .

$(3) \Rightarrow(2)$ The transitivity of $G$ implies that $D_{1}$ or $D_{5}$ are excluded, as they do not satisfy the definition of transitivity.

Observation 13 If $G \in D S C$, then the underlying undirected graph of the symmetric part of $G$ is a simple co-graph and the asymmetric part of $G$ is an oriented simple co-graph.

This holds since the asymmetric part is constructed according to the same rules as simple co-graphs and the asymmetric part with the rules of OSC.

\subsection{Directed co-simple co-graphs}

We introduce the complementary class of DSC since it holds that

$$
\begin{aligned}
& \left\{D_{1}, \ldots, D_{8}, Q_{1}, \ldots, Q_{7}, \text { co- } D_{9}, \text { co- } D_{10}, \text { co- } D_{11}\right\} \\
& \quad \neq \text { co- }\left\{D_{1}, \ldots, D_{8}, Q_{1}, \ldots, Q_{7}, \text { co- } D_{9}, \text { co- } D_{10}, \text { co- } D_{11}\right\} .
\end{aligned}
$$

Definition 15 (Directed co-simple co-graphs) The class of directed co-simple cographs is recursively defined as follows.

(i) Every digraph on a single vertex $(\{v\}, \emptyset)$, denoted by $\bullet$, is a directed co-simple co-graph. 
(ii) If $G$ is a directed co-simple co-graph and $K$ is a bidirectional complete digraph, then (a) $G \oplus K$, (b) $G \oslash K$, (c) $K \oslash G$, and (d) $G \otimes K$ are directed co-simple co-graphs.

We denote the class of directed simple co-graphs by DCSC.

Theorem 17 The following statements are equivalent.

1. $G \in D C S C$.

2. $G \in$ Free $\left(\left\{D_{1}, \ldots, D_{8}\right.\right.$, co- $\left.\left.Q_{1}, \ldots, c o-Q_{7}, Q_{1}, D_{9}, D_{10}\right\}\right)$.

3. $G \in$ Free $\left(\left\{D_{1}, \ldots, D_{8}\right.\right.$, co- $Q_{1}$, co- $Q_{4}, \ldots$, co- $\left.\left.Q_{6}, Q_{1}, D_{10}\right\}\right)$ and und $(G)$ is a co-simple co-graph.

Proof By Lemma 1, we know that co-DSC $=$ Free $\left(\right.$ co- $\left\{D_{1}, \ldots, D_{8}, Q_{1}, \ldots, Q_{7}\right.$, co- $D_{9}$, co- $D_{10}$, co- $\left.\left.D_{11}\right\}\right)$. Since DCSC $=$ co-DSC, we know by Theorem 15 that the forbidden induced subdigraphs are $D_{1}, \ldots, D_{8}$ since, they are self complementary, and co- $Q_{1}, \ldots$, co- $Q_{7}, Q_{1}, D_{9}, D_{10} \cdot{ }^{9}$ Consequently, this equivalence holds.

\subsection{Oriented co-simple co-graphs}

Definition 16 (Oriented co-simple co-graphs) The class of oriented co-simple cographs is recursively defined as follows.

(i) Every digraph on a single vertex $(\{v\}, \emptyset)$, denoted by $\bullet$, is an oriented co-simple co-graph.

(ii) If $G$ is an orientated co-simple co-graph and $T$ is a transitive tournament, then (a) $G \oplus T$, (b) $G \oslash T$, and (c) $T \oslash G$ are oriented co-simple co-graphs.

We denote the class of orientated simple co-graphs by OCSC.

It is obvious, that the classes OCSC and OCWQT are equal.

\subsection{Directed threshold graphs}

The class of threshold graphs was introduced by Chvátal and Hammer (see Chvátal and Hammer 1973, 1977). Some possible definitions of directed threshold graphs can be found in Gurski and Rehs (2019), they work as follows.

Definition 17 (Directed threshold graphs) The class of directed threshold graphs is recursively defined as follows.

(i) Every digraph on a single vertex $(\{v\}, \emptyset)$, denoted by $\bullet$, is a directed threshold graph.

(ii) If $G$ is a directed threshold graph, then (a) $G \oplus \bullet$, (b) $G \oslash \bullet$, (c) $\bullet \oslash G$, and (d) $G \otimes \bullet$ are directed threshold graphs.

We denote the class of directed threshold graphs by DT.

\footnotetext{
${ }^{9}$ Note that $Q_{1}=D_{11}$.
} 
The recursive definition of directed and undirected threshold graphs lead to the following observation.

Observation 14 For every directed threshold graph $G$ the underlying undirected graph und $(G)$ is a threshold graph.

There are also several other ways to define directed threshold graphs. Not only by forbidden induced subdigraphs, but also by graph parameters as directed linear NLC-width and directed neighbourhood width (see Gurski and Rehs 2019) for their definition.

Theorem 18 Let $G$ be a digraph. The following properties are equivalent:

1. $G$ is a directed threshold graph.

2. $G \in$ Free $\left(\left\{D_{1}, \ldots, D_{15}\right.\right.$, co- $D_{11}$, co- $D_{10}$, co- $\left.\left.D_{9}\right\}\right)$.

3. $G \in$ Free $\left(\left\{D_{1}, D_{2}, D_{3}, D_{4}, D_{5}, D_{6}, D_{10}, D_{11}, D_{13}, D_{14}, D_{15}\right\}\right)$ and und $(G) \in$ Free $\left(\left\{P_{4}, 2 K_{2}, C_{4}\right\}\right)$.

4. $G \in$ Free $\left(\left\{D_{1}, D_{2}, D_{3}, D_{4}, D_{5}, D_{6}, D_{10}, D_{11}, D_{13}, D_{14}, D_{15}\right\}\right)$ and und $(G)$ is a threshold graph.

5. G has directed linear NLC-width 1.

6. G has directed neighbourhood-width 1.

7. $G$ has directed linear clique-width at most 2 and $G \in \operatorname{Free}\left(\left\{D_{2}, D_{3}, D_{9}, D_{10}\right.\right.$, $\left.\left.D_{12}, D_{13}, D_{14}\right\}\right)$.

8. $G$ and co- $G$ are both directed trivially perfect graphs.

Proof (1) $\Rightarrow$ (2) The given forbidden graphs are not directed threshold graphs and the set of directed threshold graphs is closed under taking induced subdigraphs.

$(2) \Rightarrow$ (1) If digraph $G$ does not contain $D_{1}, D_{2}, D_{3}, D_{4}, D_{5}, D_{6}, D_{7}$ or $D_{8}$ (see Table 3) digraph $G$ is a directed co-graph by Crespelle and Paul (2006) and thus, can be constructed by using disjoint union, series composition, and order composition. By excluding $D_{9}, D_{10}$, and $D_{11}$ we know that for every series composition of $G_{1}$ and $G_{2}$ either $G_{1}$ or $G_{2}$ is bidirectional complete. Consequently, this subdigraph can also be added by a number of series operations with one vertex.

Further, by excluding $D_{12}, D_{13}, D_{14}$, and $D_{15}$ we know that for every order composition of $G_{1}$ and $G_{2}$ either $G_{1}$ or $G_{2}$ is a tournament and since we exclude a directed cycle of length 3 by $D_{5}$, by Lemma 5 we know that $G_{1}$ or $G_{2}$ is a transitive tournament. Thus, this subdigraph can also be added by a number of order operations with one vertex.

By excluding co- $D_{11}$, co- $D_{10}$, co- $D_{9}$ for every disjoint union of $G_{1}$ and $G_{2}$ either $G_{1}$ or $G_{2}$ has no edges. Thus, this subdigraph can also be added by a number of disjoint union operations with one vertex.

(3) $\Leftrightarrow$ (4) Since Forb(T) $=\left\{C_{4}, P_{4}, 2 K_{2}\right\}$.

(1) $\Leftrightarrow(5)$ and (5) $\Leftrightarrow$ (6) (Gurski and Rehs 2019).

(1) $\Rightarrow$ (7) and (7) $\Rightarrow$ (2) (Gurski and Rehs 2019).

(2) $\Rightarrow$ (4) By Observation 14.

(3) $\Rightarrow$ (2) By Observation 2.

$(1) \Rightarrow(8) G$ and co- $G$ are both directed threshold graphs and thus, both are directed trivially perfect graphs. 
$(8) \Rightarrow(1)$ Let $X=\left\{D_{1}, \ldots, D_{15}\right\}$. By Theorem 7 we know that $G \in \operatorname{Free}(X)$ and co- $G \in$ Free $(X)$ hold. Lemma 1 implies that $G \in \operatorname{Free}(X) \cap$ Free $($ co- $X)$. And again by Lemma 1 we obtain $G \in \operatorname{Free}(X \cup \operatorname{co}-X)$. By Observation 4 and part (2) of this theorem it holds that $G$ is a directed threshold graph.

For directed threshold graphs Observation 4 leads to the next result.

Proposition $3 D T=c o-D T$

Similar as undirected threshold graphs (cf. Hagberg et al. 2006), directed threshold graphs can also be characterized by the existence of a special sequence.

A directed creation sequence for $G=(V, E)$ with $V=\left\{v_{1}, \ldots, v_{n}\right\}$ is a quaternary string $t=t_{1}, \ldots, t_{n}$ of length $n$ such that there is a bijection $v:\{1, \ldots, n\} \rightarrow V$ with

$-t_{i}=3$ if $v(i)$ is a bi-dominating vertex for the graph $G[\{v(1), \ldots, v(i)\}]$

$t_{i}=2$ if $v(i)$ is a in-dominating vertex for the graph $G[\{v(1), \ldots, v(i)\}]$

$-t_{i}=1$ if $v(i)$ is a out-dominating vertex for the graph $G[\{v(1), \ldots, v(i)\}]$ and

$-t_{i}=0$ if $v(i)$ is an isolated vertex for the graph $G[\{v(1), \ldots, v(i)\}]$.

W.l.o.g. we define a single vertex to be a dominating vertex, i.e. $t_{1}=1$. By adapting the result for undirected threshold graphs from Mahadev and Peled (1995, Fig. 1.4) a directed creation sequence can be found in time $\mathcal{O}(n+m)$. Furthermore this implies that directed threshold graphs can be recognized in time $\mathcal{O}(n+m)$.

\subsection{Oriented threshold graphs}

The class of oriented threshold graphs has been introduced in Boeckner (2018) as follows.

Definition 18 (Oriented threshold graphs Boeckner 2018) A graph $G=(V, A)$ is an threshold graph if there exists an injective weight function $w: V \rightarrow \mathbb{R}$ and a threshold value $t \in \mathbb{R}$ such that $(x, y) \in A$ if and only if $|w(x)|+|w(y)| \geq t$ and $w(x)>w(y)$.

Theorem 19 (Boeckner 2018) Let $G$ be an oriented digraph. The following properties are equivalent:

1. $G$ is an oriented threshold graph.

2. $G \in \operatorname{Free}\left(\left\{D_{1}, D_{5}\right\}\right)$ and und $(G) \in \operatorname{Free}\left(\left\{2 K_{2}, C_{4}, P_{4}\right\}\right)$.

3. $G$ is a transitive orientation of a threshold graph.

4. G can be constructed from the one vertex empty graph by successively adding an isolated vertex, an out-dominating vertex or an in-dominated vertex.

Using Theorem 19 we obtain the following definition, which is equivalent to Definition 18:

Definition 19 (Oriented threshold graphs) The class of oriented threshold graphs is recursively defined as follows.

(i) Every digraph on a single vertex $(\{v\}, \emptyset)$, denoted by $\bullet$, is an oriented threshold graph. 
(ii) If $G$ is an oriented threshold graph, then (a) $G \oplus \bullet$, (b) $G \oslash \bullet$, and (c) $\bullet \oslash$ are oriented threshold graphs.

We denote the class of oriented threshold graphs by OT.

The recursive definition of oriented and undirected threshold graphs lead to the following observation.

Observation 15 For every oriented threshold graph $G$ the underlying undirected graph und $(G)$ is a co-graph.

This class can also be defined by forbidden induced subdigraphs. As it was possible for oriented co-graphs and oriented trivially perfect graphs, we can use the fact that oriented threshold graphs are exactly the directed threshold graphs not containing an induced $\overleftrightarrow{K_{2}}$ :

Theorem 20 Let $G$ be a digraph. The following properties are equivalent:

1. G is an oriented threshold graph.

2. $G$ is an oriented co-trivially perfect graph.

3. $G \in$ Free $\left(\left\{D_{1}, D_{5}, D_{8}, D_{12}, 2 \overrightarrow{P_{2}}, \overleftrightarrow{K_{2}}\right\}\right)$.

4. $G \in$ Free $\left(\left\{D_{1}, D_{5}, \overleftrightarrow{K_{2}}\right\}\right)$ and und $(G) \in$ Free $\left(\left\{2 K_{2}, C_{4}, P_{4}\right\}\right)$.

5. $G \in$ Free $\left(\left\{D_{1}, D_{5}, \overleftrightarrow{K_{2}}\right\}\right)$ and und $(G)$ is a threshold graph.

6. $G \in$ Free $\left(\left\{D_{1}, D_{5}, D_{12}, \overleftrightarrow{K_{2}}\right\}\right)$ and und $(G) \in$ Free $\left(\left\{P_{4}, 2 K_{2}\right\}\right)$.

7. $G \in$ Free $\left(\left\{D_{1}, D_{5}, D_{12}, \overleftrightarrow{K_{2}}\right\}\right)$ and und $(G)$ is a co-trivially perfect graph.

8. $G \in$ Free $\left(\left\{D_{1}, D_{5}, D_{12}, 2 \overrightarrow{P_{2}}, \overleftrightarrow{K_{2}}\right\}\right)$ and und $(G) \in$ Free $\left(\left\{P_{4}\right\}\right)$.

$9 G \in \operatorname{Free}\left(\left\{D_{1}, D_{5}, D_{12}, 2 \overrightarrow{P_{2}}, \overleftrightarrow{K_{2}}\right\}\right)$ and und $(G)$ is a co-graph.

10. $G$ is transitive and $G \in$ Free $\left(\left\{D_{8}, D_{12}, 2 \overrightarrow{P_{2}}, \overleftrightarrow{K_{2}}\right\}\right)$.

Proof $(1) \Leftrightarrow(2)$ By the recursive definition of the classes, which arises from the restriction of the directed classes to oriented graphs.

$(1) \Rightarrow(3)$ If $G$ is an oriented threshold graph, then $G$ is a directed threshold graph and by Theorem 18 it holds that $G \in$ Free $\left(\left\{D_{1}, \ldots, D_{15}\right.\right.$, co- $D_{11}$, co- $D_{10}$, co- $\left.\left.D_{9}\right\}\right)$. Further $G \in \operatorname{Free}\left(\left\{\overleftrightarrow{K_{2}}\right\}\right)$ because of the missing series composition. This leads to $G \in \operatorname{Free}\left(\left\{D_{1}, D_{5}, D_{8}, D_{12}, \overleftrightarrow{K_{2}}, 2 \vec{P}_{2}\right\}\right)$

(3) $\Rightarrow$ (1) If $G \in \operatorname{Free}\left(\left\{D_{1}, D_{5}, D_{8}, D_{12}, \overleftrightarrow{K_{2}}, 2 \vec{P}_{2}\right\}\right)$, then $G \in \operatorname{Free}\left(\left\{D_{1}, \ldots\right.\right.$, $D_{15}$, co- $D_{11}$, co- $D_{10}$, co- $\left.\left.D_{9}\right\}\right)$ and is a directed threshold graph. Since $G \in$ Free $\left(\left\{\overleftrightarrow{K_{2}}\right\}\right)$ there is no series operation in any construction of $G$ which implies that $G$ is an oriented threshold graph.

(4) $\Leftrightarrow(5)$ Since Forb(T) $=\left\{C_{4}, P_{4}, 2 K_{2}\right\}$.

(6) $\Leftrightarrow$ (7) Since Forb (CTP) $=\left\{P_{4}, 2 K_{2}\right\}$.

$(8) \Leftrightarrow(9)$ Since Forb $(C)=\left\{P_{4}\right\}$.

(3) $\Rightarrow(5),(3) \Rightarrow$ (7), and (3) $\Rightarrow$ (9) By Observation 15.

(4) $\Rightarrow(3),(6) \Rightarrow$ (3), and (8) $\Rightarrow$ (3) By Observation 2.

(3) $\Rightarrow$ (10) By Lemma 3 we know that $G$ is transitive.

$(10) \Rightarrow$ (3) If $G$ is transitive, then $G \in \operatorname{Free}\left(\left\{D_{1}, D_{5}\right\}\right)$. 
Fig. 2 A 2-switch. All vertices are distinct. Solid arcs must appear in the digraph and dashed arcs must not appear in the digraph. If an arc is not given, then it may or may not be present

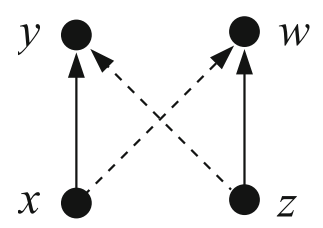

A very famous subclass of oriented threshold graphs is the class of transitive tournaments, which can be easily constructed by repeating $G \oslash \bullet$.

The proof for the next Theorem can be done very similar to the proof of Theorem 10 .

Theorem 21 A graph $G$ is a threshold graph if and only if there exists an orientation $G^{\prime}$ of $G$ such that $G^{\prime}$ is an oriented threshold graph.

Observation 16 If $G \in D T$, then the underlying undirected graph of the symmetric part of $G$ is a threshold graph and the asymmetric part of $G$ is an oriented threshold graph.

This holds since the asymmetric part is constructed according to the same rules as threshold graphs and the asymmetric part is constructed according to the rules of OT.

Similar to directed threshold graphs every oriented co-graph can be defined by a sequence with only three operations, which can be used to give a linear time recognition algorithm.

\subsection{Threshold digraphs and Ferres digraphs}

The following idea of defining a directed version of threshold graphs, i.e. threshold digraphs, is from Cloteaux et al. (2014) where they define them by a set of forbidden subdigraphs.

A 2 -switch is a vertex set $\{w, x, y, z\}$ such that there exist the edges $(w, x)$ and $(y, z)$ but not the edges $(w, z)$ and $(y, x)$, see Fig. 2. Examples for a 2-switch are co- $D_{10}$, co- $D_{9}$, co- $D_{11}$ and $\vec{P}_{4}$.

Observation 17 Let $G$ be a directed threshold graph, then $G$ does not contain a 2switch.

Definition 20 (Threshold digraphs Cloteaux et al. 2014) A digraph $G$ is a threshold digraph if it does not contain a 2 -switch nor a $D_{5}$ as induced subdigraph.

The class of threshold digraphs is denoted by TD.

This class is not very useful for our directed co-graph hierarchy, as they are incomparable to most of the graph classes in it, though it is a superclass of directed threshold graphs (see Sect. 4.17).

A well studied class of digraphs are Ferres digraphs, see (Mahadev and Peled 1995, Chapter 2) for a survey. Ferres digraphs are introduced by Riguet in Riguet (1951). In their first definition, Ferres digraphs were defined on directed graphs including loops. As our subclasses of directed co-graphs do not use loops, only Ferres digraphs without 
Fig. 3 An alternating

4-anticircuit. The vertices are not necessarily distinct but $x \neq z$ and $y \neq w$. The solid arrows represent the presence of an arc and a dashed arrow its absence

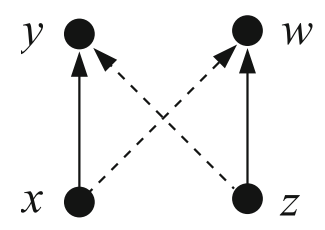

loops will be used here. An alternating 4-anticircuit consists of vertices $x, y, z, w$, not necessarily distinct but $x \neq z$ and $y \neq w$, satisfying $(x, y),(z, w) \in A$ and $(x, w),(z, y) \notin A$ (cf. Fig. 3).

Definition 21 (Ferres digraphs) A digraph is a Ferres digraph if it does not contain an alternating 4-anticircuit.

The class of Ferres digraphs is denoted by FD.

By considering all possible equalities of vertices in an alternating 4-anticircuit, an equivalent characterization is obtained by $G \in \operatorname{Free}\left(\left\{D_{1}, \overleftrightarrow{K_{2}}\right\}\right)$ and $G$ does not contain a 2-switch, see (Mahadev and Peled 1995, Figure 2.2) additional to the restriction to digraphs without loops. This class is comparable to oriented threshold graphs, but not to any other graph class in the directed co-graph hierarchy, as we will see in Sect. 4.18.

\subsection{Overview}

In Table 14 we summarize directed co-graphs and their subclasses.

Since directed co-graphs and all defined subclasses are hereditary, by Theorem 2 there exist sets of minimal forbidden induced subdigraphs. The given theorems even show this finite sets of minimal forbidden induced subdigraphs for the different classes. These characterizations lead to polynomial time recognition algorithms for the corresponding graph classes.

\subsection{Relations}

Our forbidden subdigraphs in combination with Theorem 3 allows us to compare the above graph classes to each other and show the hierarchy of the subclasses of directed co-graphs, see Fig. 4.

\section{Conclusions and outlook}

We introduced several new digraph classes. All of these classes are subsets of directed co-graphs which have been defined by Bechet et al. (1997) and supersets of oriented threshold graphs defined by Boeckner (2018). Additionally, we consider the ideas of Cloteaux et al. (2014) and Ferres digraphs (Riguet 1951).

Our characterizations by final sets of forbidden induced subdigraphs lead to polynomial time recognition algorithms for the corresponding graph classes. In Sects. 4.14 and 4.15 we suggested linear time methods for the recognition of directed and oriented 


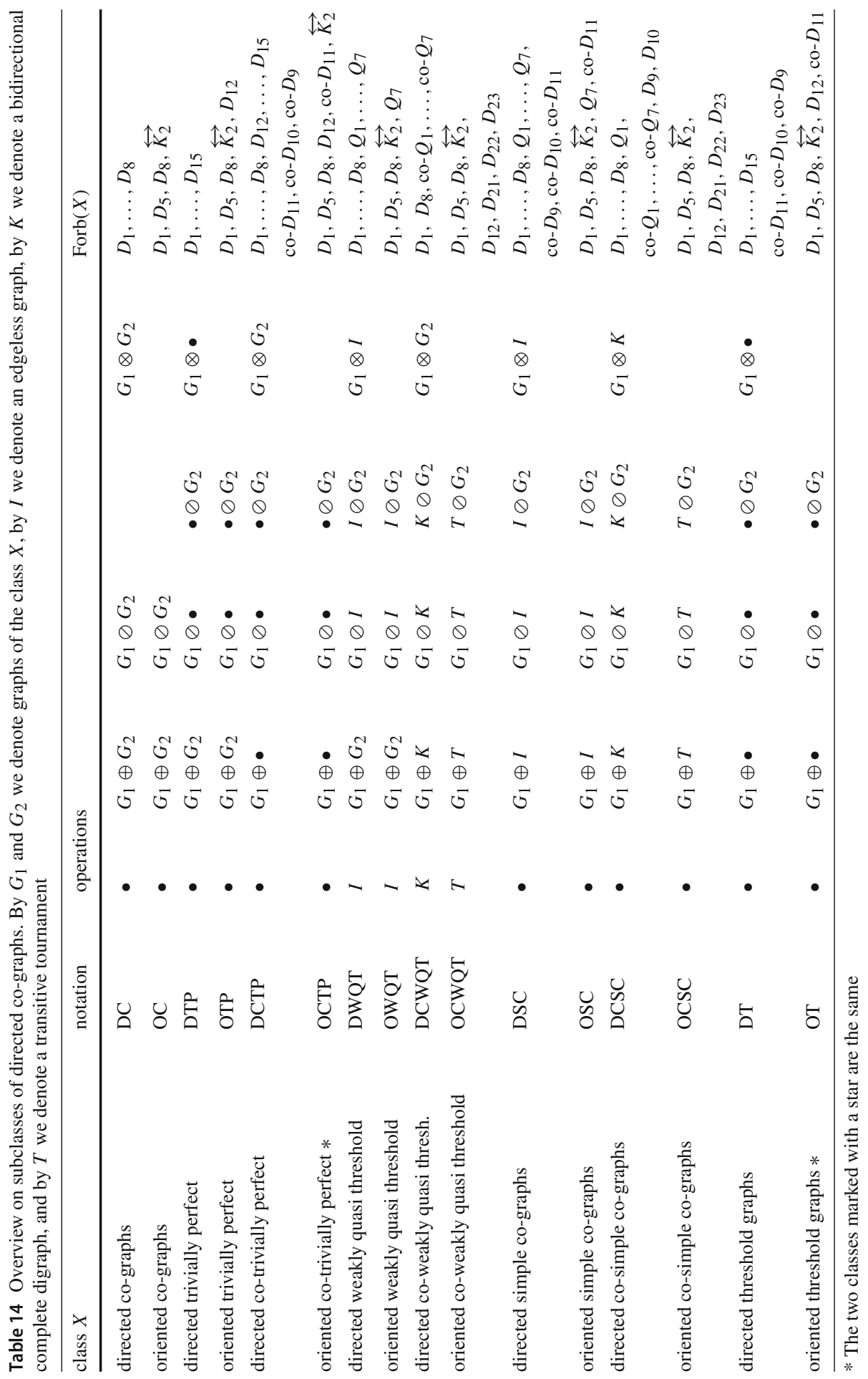




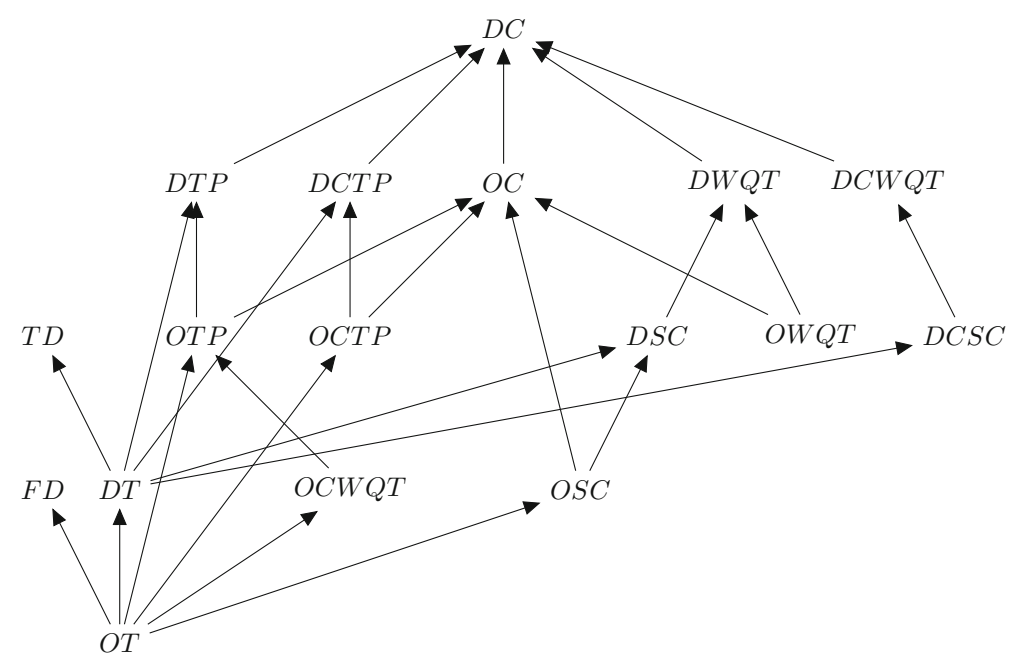

Fig. 4 Relations between the subclasses of directed co-graphs. If there is a path from $A$ to $B$, then it holds that $A \subset B$. The classes, that are not connected by a directed path are incomparable

threshold graphs. For the other classes it remains open to find more efficient algorithms for this purpose.

For directed co-graphs we have shown in Gurski and Rehs (2018b) that the directed path-width equals to the directed tree-width and how to compute this value in linear time. Moreover, the digraph width parameters directed feedback vertex set number, cycle rank, DAG-depth, and DAG-width can be computed in linear time for directed co-graphs (Gurski et al. 2019b). It remains to verify the relation of these parameters restricted to threshold digraphs and Ferres digraphs.

In Johnson et al. (2001) the directed union was introduced as a generalization of the disjoint union and the order composition. In Gurski and Rehs (2018a) we considered digraphs which can be defined by disjoint union, order composition, directed union, and series composition of two directed graphs. The set of all these digraphs is denoted by extended directed co-graphs since it generalizes directed co-graphs. In Gurski and Rehs (2018a) we showed that the result of Gurski and Rehs (2018b) even holds for extended directed co-graphs. For the class of extended directed co-graphs it remains to show how to compute an ex-di-co-tree and to find forbidden subdigraph characterizations. Furthermore, it also has a number of interesting subclasses beside those given in this paper.

Acknowledgements This work was funded in part by the Deutsche Forschungsgemeinschaft (DFG, German Research Foundation) - 388221852

Funding Open Access funding enabled and organized by Projekt DEAL.

Data Availability Statement Our manuscript has no associated data.

Open Access This article is licensed under a Creative Commons Attribution 4.0 International License, which permits use, sharing, adaptation, distribution and reproduction in any medium or format, as long as you give 
appropriate credit to the original author(s) and the source, provide a link to the Creative Commons licence, and indicate if changes were made. The images or other third party material in this article are included in the article's Creative Commons licence, unless indicated otherwise in a credit line to the material. If material is not included in the article's Creative Commons licence and your intended use is not permitted by statutory regulation or exceeds the permitted use, you will need to obtain permission directly from the copyright holder. To view a copy of this licence, visit http://creativecommons.org/licenses/by/4.0/.

\section{References}

Bechet D, de Groote P, Retoré C (1997) A complete axiomatisation of the inclusion of series-parallel partial orders. In: Rewriting techniques and applications, LNCS, vol 1232. Springer, pp 230-240

Bang-Jensen J, Gutin G (2009) Digraphs. Theory, algorithms and applications. Springer, Berlin

Bang-Jensen J, Gutin G (eds) (2018) Classes of directed graphs. Springer, Berlin

Bang-Jensen J, Maddaloni A (2014) Arc-disjoint paths in decomposable digraphs. J Graph Theory 77:89110

Bapat RB, Lal AK, Pati S (2008) Laplacian spectrum of weakly quasi-threshold graphs. Graphs Comb 24(4):273-290

Bodlaender HL, Möhring RH (1993) The pathwidth and treewidth of cographs. SIAM J. Disc. Math. 6(2):181-188

Boeckner D (2018) Oriented threshold graphs. Austral J Comb 71(1):43-53

Chvátal V, Hammer PL (1973) Set-packing and threshold graphs. Technical Report CORR 73-21, Computer Science Department, University of Waterloo

Chvátal V, Hammer PL (1977) Aggregation of inequalities in integer programming. Ann Discrete Math $1: 145-162$

Cloteaux B, LaMar MD, Moseman E, Shook J (2014) Threshold Digraphs. J Res Natl Inst Stand Technol 119:227-234

Corneil DG, Lerchs H, Stewart-Burlingham L (1981) Complement reducible graphs. Discrete Appl Math $3: 163-174$

Courcelle B, Olariu S (2000) Upper bounds to the clique width of graphs. Discrete Appl Math 101:77-114

Crespelle C, Paul C (2006) Fully dynamic recognition algorithm and certificate for directed cographs. Discrete Appl Math 154(12):1722-1741

Corneil DG, Perl Y, Stewart LK (1984) Cographs: recognition, applications, and algorithms. In: Proceedings of 15 th southeastern conference on combinatorics, graph theory, and computing

Gurski F, Hoffmann S, Komander D, Rehs C, Rethmann J, Wanke E (2020a) Computing directed Steiner path covers for directed co-graphs. In: Proceedings of the conference on current trends in theory and practice of computer science (SOFSEM), LNCS, vol 12011. Springer, pp 556-565

Gurski F, Hoffmann S, Komander D, Rehs C, Rethmann J, Wanke E (2020b) Exact solutions for the Steiner path problem on special graph classes. In: Operations research proceedings (OR 2019), selected papers. Springer, pp 331-338

Gurski F, Komander D, Lindemann M (2020c) Oriented coloring of msp-digraphs and oriented co-graphs. In: Proceedings of the international conference on combinatorial optimization and applications (COCOA), LNCS. Springer (to appear)

Gurski F, Komander D, Rehs C (2019) Characterizations for special directed co-graphs. In: Proceedings of the international conference on combinatorial optimization and applications (COCOA), LNCS, vol 11949. Springer, pp 252-264

Gurski F, Komander D, Rehs C (2019a) Computing digraph width measures on directed co-graphs. In: Proceedings of international symposium on fundamentals of computation theory (FCT), LNCS, vol 11651. Springer, pp 292-305

Gurski F, Komander D, Rehs C (2019c) Oriented coloring on recursively defined digraphs. Algorithms 12(4):87

Golumbic MC (1978) Trivially perfect graphs. Discrete Math 24:105-107

Golumbic MC (1980) Algorithmic graph theory and perfect graphs. Academic Press, London

Gould R (2012) Graph theory. Dover, New York

Gurski F, Gurski and Rehs C (2018a) Computing directed path-width and directed tree-width of recursively defined digraphs. ACM Computing Research Repository (CoRR). arXiv:1806.04457 
Gurski F, Rehs C (2018b) Directed path-width and directed tree-width of directed co-graphs. In: Proceedings of the international conference on computing and combinatorics (COCOON), LNCS, vol 10976. Springer, pp 255-267

Gurski F, Rehs C (2019) Comparing linear width parameters for directed graphs. Theory Comput Syst 63(6):1358-1387

Gurski F, Rehs C, Rethmann J (2018) Directed pathwidth of sequence digraphs. In: Proceedings of the international conference on combinatorial optimization and applications (COCOA), LNCS, vol 11346. Springer, pp 79-93

Gurski F (2017) Dynamic programming algorithms on directed cographs. Stat Optim Inf Comput 5:35-44

Gurski F, Wanke E, Yilmaz E (2016) Directed NLC-width. Theor Comput Sci 616:1-17

Heggernes P, Meister D, Papadopoulos C (2011) Graphs of linear clique-width at most 3. Theor Comput Sci 412(39):5466-5486

Hagberg A, Swart PJ, Schult DA (2006) Designing threshold networks with given structural and dynamical properties. Phys Rev E 74:056116

Johnson T, Robertson N, Seymour PD, Thomas R (2001) Directed tree-width. J Comb Theory Ser B 82:138-155

Kitaev S, Lozin V (2015) Words and graphs. Springer, Berlin

Lawler EL (1976) Graphical algorithms and their complexity. Math Centre Tracts 81:3-32

Lerchs H (1971) On cliques and kernels. Technical report, Department of Computer Science, University of Toronto

Lin R, Olariu S, Pruesse G (1995) An optimal path cover algorithm for cographs. Comput Math Appl 30:75-83

Mahadev NVR, Peled UN (1995) Threshold Graphs and Related Topics. Annals of Discrete Math. 56. Elsevier, North-Holland

Nojgaard N, El-Mabrouk N, Merkle D, Wieseke N, Hellmuth M (2018) Partial homology relationssatisfiability in terms of di-cographs. In: Proceedings of international computing and combinatorics conference (COCOON), LNCS, vol 10976. Springer, pp 403-415

Nikolopoulos SD, Papadopoulos C (2011) A simple linear-time recognition algorithm for weakly quasithreshold graphs. Graphs Comb 27(4):557-565

Retoré C (1999) Pomset logic as a calculus of directed cographs. In: Fourth Roma workshop: dynamic perspectives in Logic and Linguistics. CLUEB, pp 221-247

Riguet J (1951) Les relations de ferrers. CR Acad Sci Paris 232:1729-1730

Sumner PD (1974) Dacey graphs. J Aust Soc 18:492-502

Valdes J, Tarjan RE, Lawler EL (1982) The recognition of series-parallel digraphs. SIAM J Comput 11:298313

Publisher's Note Springer Nature remains neutral with regard to jurisdictional claims in published maps and institutional affiliations. 